\title{
Late Pleistocene-Holocene radiolarian paleotemperatures in the Norwegian Sea based on artificial neural networks
}

\author{
Giuseppe Cortese $^{\mathrm{a}, *}$, Jane K. Dolven ${ }^{\mathrm{b}}$, Kjell R. Bjørklund ${ }^{\mathrm{b}}$, Björn A. Malmgren ${ }^{\mathrm{c}}$ \\ ${ }^{a}$ Alfred Wegener Institute for Polar and Marine Research (AWI), Columbusstrasse, P.O. Box 120161-27515 Bremerhaven, Germany \\ ${ }^{\mathrm{b}}$ Geological Museum, University of Oslo, P.O. Box 1172 Blindern, 0318 Oslo, Norway \\ ${ }^{c}$ Department of Earth Sciences-Marine Geology, Göteborg University, Box 460, SE-405 30 Göteborg, Sweden
}

Received 19 May 2004; received in revised form 13 April 2005; accepted 14 April 2005

\begin{abstract}
Artificial Neural Networks (ANN) were trained by using an extensive radiolarian census dataset from the Nordic (Greenland, Norwegian, and Iceland) Seas. The regressions between observed and predicted Summer Sea Temperature (SST) indicate that lower error margins and better correlation coefficients are obtained for $100 \mathrm{~m}\left(\mathrm{SST}_{100}\right)$ compared to $10 \mathrm{~m}\left(\mathrm{SST}_{10}\right)$ water depth, and by using a subset of species instead of all species. The trained ANNs were subsequently applied to radiolarian data from two Norwegian Sea cores, HM 79-4 and MD95-2011, for reconstructions of SSTs through the last 15,000 years. The reconstructed SST is quite high during the Bølling-Allerød, when it reaches values only found later during the warmest phase of the Holocene. The climatic transitions in and out of the Younger Dryas are very rapid and involve a change in $\mathrm{SST}_{100}$ of 6.2 and $6.8{ }^{\circ} \mathrm{C}$, taking place over 440 and 140 years, respectively. $\mathrm{SST}_{100}$ remains at a maximum during the early Holocene, and this Radiolarian Holocene Optimum Temperature Interval (RHOTI) predates the commonly recognized middle Holocene Climatic Optimum (HCO). During the $8.2 \mathrm{ka}$ event, $\mathrm{SST}_{100}$ decreases by ca. $3{ }^{\circ} \mathrm{C}$, and this episode marks the establishment of a cooling trend, roughly spanning the middle Holocene (until ca. $4.2 \mathrm{ka}$ ). Successively, since then and through the late Holocene, $\mathrm{SST}_{100}$ follows instead a statistically significant warming trend. The general patterns of the reconstructed SSTs agree quite well with previously obtained results based on application of Imbrie and Kipp Transfer Functions (IKTF) to the same two cores for $\mathrm{SST}_{0}$. A statistically significant cyclic component of our SST record (period of 278 years) has been recognized. This is close to the de Vries or Suess cycle, linked to solar variability, and documented in a variety of other high-resolution Holocene records. (C) 2005 Elsevier B.V. All rights reserved.
\end{abstract}

Keywords: Artificial neural networks; Radiolarians; Nordic seas; Late Pleistocene; Holocene

* Corresponding author. Tel.: +49 4714831 1207; fax: +49 471 48311149.

E-mail address: gcortese@awi-bremerhaven.de (G. Cortese).

\section{Introduction}

The Nordic Seas, together with the Labrador Sea, represent today the main site of convection for the deep waters alimenting the North Atlantic Deep 
Water, i.e. the deep return flow of the large-scale overturning circulation. The environmental variables and processes linked to deep-water formation in this area (temperature, salinity and density characteristics of the incoming surface waters, heat release to the atmosphere, sea-ice extent, brine formation) exert a direct control on regional and hemispheric climate. In fact, the thermohaline overturning circulation regulates the heat balance at these high northern latitudes, and during past climate cycles it has shown different equilibrium states (Ganopolski and Rahmstorf, 2001). As these have a direct impact on the climate of surrounding landmasses, it is of interest to reconstruct the changes in the temperature of the main surface water body (i.e. the Norwegian Current) in this climatically sensible area over the Late Pleistocene-Holocene interval, including the last deglaciation.

A broad range of different statistical methods has been used, during the past few decades, to reconstruct paleoclimate. They range from Imbrie and Kipp Transfer Functions (IKTF) (Imbrie and Kipp, 1971) to Canonical Correspondence Analysis (ter Braak, 1986), Modern Analog Technique (MAT) (Hutson, 1979), modern analog with similarity index method (SIMMAX) (Pflaumann et al., 1996), the Revised Analog Method (RAM) (Waelbroeck et al., 1998), Weighted Averaging Partial Least Squares (WAPLS) (ter Braak and Juggins, 1993; Birks, 1995), and Artificial Neural Networks (ANN) (McCulloch and Pitts, 1943; Malmgren and Nordlund, 1997).

Several of these techniques were successfully used to extract paleoclimatic information from census data based on different microfossil groups. IKTF has been applied to radiolarians from the Pacific Ocean (Moore, 1973; Molina-Cruz, 1984; Pisias et al., 1997), the Southern Ocean (Abelmann et al., 1999; Cortese and Abelmann, 2002), the Atlantic Ocean (Morley, 1979), and the Nordic Seas (Bjørklund et al., 1998; Dolven et al., 2002; Cortese et al., 2003). In this paper, ANNs were trained on the basis of radiolarian relative abundance data from surface sediment samples collected in the Nordic Seas, and the trained ANNs were applied to high-resolution down-core records from the eastern Norwegian Sea (cores HM79-4 and MD95-2011). We will present the main results obtained from the network calibration phase, and the obtained $\mathrm{SST}_{100}$ (Summer Sea Temperature at
$100 \mathrm{~m}$ ) estimates. The down-core paleotemperature predictions based on ANN will be discussed in relation to other Late Pleistocene-Holocene paleoclimatic records.

\section{Methods}

\section{1. "Artificial" neural networks and statistical testing procedures}

Artificial Neural Networks (ANN) are "selfadjusting" computer systems that can "learn" by continuously going back and changing a set of parameters in the model to reduce the error between a desired output and an actual output, i.e. in our case finding the optimal model for reconstructing $\mathrm{SST}_{100}$ from a given dataset. Our ANNs were trained on the basis of radiolarian census data collected from 161 surface sediment samples (Fig. 1, inset) from the Nordic Seas (Cortese et al., 2003). In order to reconstruct paleotemperatures, the ANNs were then applied to downcore radiolarian census data from two cores, HM79-4 and MD95-2011 (Dolven et al., 2002), sampled in the eastern Norwegian Sea (Fig. 1). Two different ANN programs, the NeuroGenetic Optimizer (NGO, version 2.5; (CBioComp Systems, Inc.) and the iModel (version 1.5, CBioComp Systems, Inc.), were initially used to assess which of them worked best. We found, based on three test runs, that the NGO generated slightly lower error rates than the iModel, so we used the NGO software in the ensuing analyses. We used the Root-Mean-Square Error of Prediction (RMSEP), which is the square root of the sum of the squared differences between the observed and predicted values for all observations in the test set divided by the number of such observations, to estimate the prediction error. We also made three separate training runs for 10 and $100 \mathrm{~m}$ water depth using the NGO, in order to assess the lowest RMSEP (Table 1), and found that the reconstruction of temperatures at $100 \mathrm{~m}$ provides best results (average RMSEP $=0.77$; average $r=0.96$ ).

In our analyses, the original data were automatically subdivided by the software into a training and a test set using a random procedure. For the 161 surface sediment samples included in the dataset, 120 samples were used as the modelling/training set, and the 


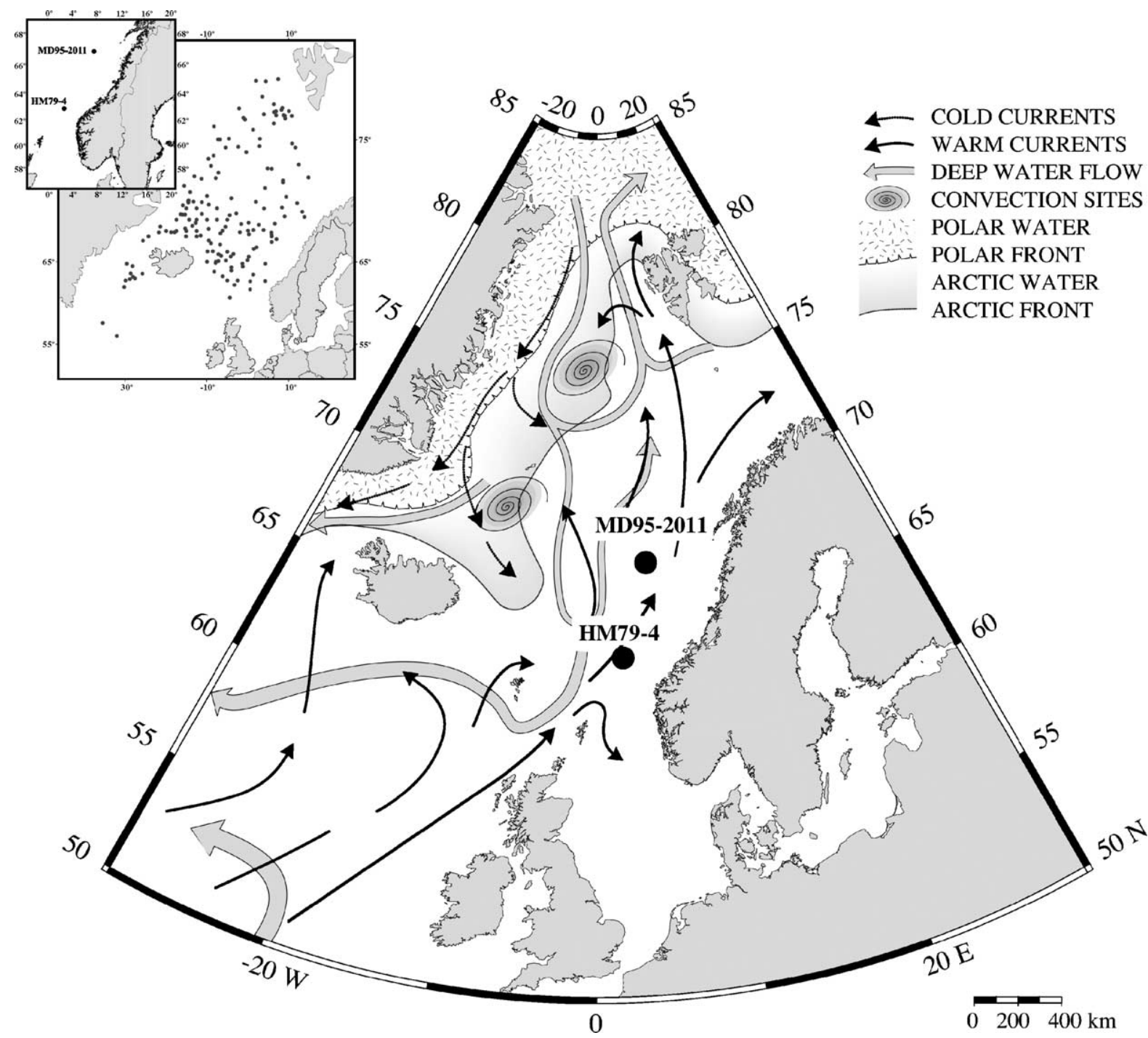

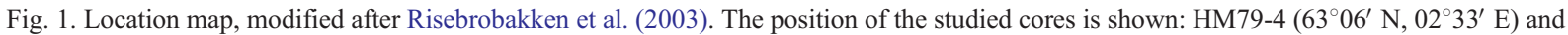
MD95-2011 (66 $58^{\prime} \mathrm{N}, 07^{\circ} 38^{\prime}$ E). The main surface and deep currents, as well as other important oceanographic features (Polar and Arctic Front, deep-water convection sites) are reported too. The inset displays the 161 surface sediment samples used for the calibration to modern hydrography conditions (Cortese et al., 2003).

remaining 41 samples as the test set. As the randomly picked test-set samples are not included in the training process, the error rates are based on this set. They therefore provide estimates of the prediction error rate in samples different than those used in the training set. In other words, the RMSEP represents an estimate of the minimum error we can expect when using the trained ANNs to "predict" past SSTs. Obtaining an RMSEP of 1.0, if the estimated SST is $7{ }^{\circ} \mathrm{C}$, means that the estimated error in paleo-SSTs predictions is at least $1{ }^{\circ} \mathrm{C}$, i.e. $7 \pm 1{ }^{\circ} \mathrm{C}$. In this sense, low RMSEP are equivalent to being able to generate past SSTs with a low error.

In tests for trend in timeseries, the confidence interval for the slope coefficient of the regression function can only be used in cases where the sequence of residuals is random and independent (uncorrelated), with residuals conforming to the normal 
Table 1

Summary of NGO training runs

\begin{tabular}{|c|c|c|c|c|c|}
\hline \multicolumn{3}{|c|}{ NGO all species } & \multicolumn{3}{|c|}{ NGO subset } \\
\hline & RMSEP & $r$ & & RMSEP & $r$ \\
\hline \multicolumn{6}{|l|}{$10 \mathrm{~m}$} \\
\hline Run 1 & 1.3861 & 0.8751 & Run 1 & 1.1597 & 0.9148 \\
\hline Run 2 & 1.2048 & 0.9316 & Run 2 & 0.9978 & 0.9422 \\
\hline Run 3 & 1.2753 & 0.9064 & Run 3 & 1.0484 & 0.9384 \\
\hline Mean & 1.2887 & 0.9044 & & 1.0686 & 0.9318 \\
\hline \multicolumn{6}{|l|}{$100 \mathrm{~m}$} \\
\hline Run 1 & 0.9485 & 0.9329 & Run 1 & 0.8619 & 0.9477 \\
\hline Run 2 & 0.8352 & 0.9566 & Run 2 & 0.7222 & 0.967 \\
\hline Run 3 & 0.7795 & 0.9607 & Run 3 & 0.7321 & 0.9654 \\
\hline Mean & 0.8544 & 0.9501 & & 0.7721 & 0.96 \\
\hline
\end{tabular}

Root Mean Square Error of Prediction (RMSEP) and correlation coefficient between observed and estimated SST $(r)$, along with their mean values, are shown for three different runs.

distribution. Since all of these assumptions were not met here, the Kendall test (Kendall and Ord, 1990) was used instead. We employed the REDFIT procedure, introduced by Schulz and Mudelsee (2002), to test for cyclical patterns in the generated $\mathrm{SST}_{100}$ timeseries. REDFIT is specifically adapted to unevenly spaced timeseries, and allows tests of the significance of peaks in the spectrum against a null-hypothesis of a red-noise background, which is estimated using an AR1 process. The REDFIT technique avoids the significant bias in the form of reddening of the spectrum that results from interpolations in the time domain to artificially create equal sampling intervals. The REDFIT procedure is associated with a runs test, aimed at assessing whether the spectrum is consistent or not with a red-noise model. If the $r$-value resulting from this test lies within a $5 \%$ acceptance region, an AR1 model is appropriate to characterize the record. We used a false-alarm level of $(1-1 / n) * 100 \%$, where $n$ is the number of spectral values in the timeseries (Thomson, 1990), which is the maximum spectral amplitude expected if the timeseries were generated by an AR1 process (Schulz and Mudelsee, 2002), as the lower level for the detection of non-AR1 components in the timeseries. In our application, the false-alarm level is $98.4 \%$. We used the mean of 2000 Monte Carlo simulations of the AR1 process to estimate the red-noise spectra in our applications (the over-sampling factor for the Lomb-Scargle
Fourier transform was set at 3.0 and the number of Welch-overlapped-segments with $50 \%$ overlap at 3 , and we used a Welch spectral window to reduce spectral leakage; details on the parameters can be found in Schulz and Mudelsee, 2002).

\subsection{Species selection}

We also operated a selection of taxa, as the 30 taxa that were included in any further statistical treatment (Table 2, Plates I and II) were chosen from an original species list comprising 114 taxa, most of which were identified to species level. During this screening phase (see Cortese et al., 2003, for additional details), taxa are excluded if they are intermediate/deep water dwellers (e.g. Cycladophora davisiana, Eucyrtidium calvertense); if taxonomic uncertainties could lead to lumping or wrong identification; if a dissolution bias is present (e.g. species particularly prone/resistant to dissolution). All these reasons blur the value of these taxa as SST reconstruction tools, and therefore lower the correlation coefficients attached to them, and widen the error bars. As our objective was to obtain reliable SST estimates (based on biology/ecology information) from the ANN, and not to optimize error rates, we however preferred to operate the initial species screening (the 30 pre-selected taxa) ourselves and not leave it to an automated routine. The preselection gives us the possibility to integrate into the dataset information concerning radiolarian ecology, habitat preferences (e.g. shallow vs. deep water living taxa), deviation from a simple relation to SST (taxa influenced by other environmental parameters), that cannot be assessed independently by ANN, and which will introduce a strong amount of noise to the estimates. This information comes from additional material, previous studies and published literature (Bjørklund et al., 1998; Cortese et al., 2003), and experience that is not reflected and/or included in the dataset. Within the 30 pre-selected taxa (Table 2 ), we then let the software automatically pick "subsets" of taxa as inputs to the program, and assess which species setup will produce the lowest RMSEPs. This procedure was preferred to manually adding or deleting arbitrarily one or more taxa and then training the networks over and over again, since the ANN procedure is quite time-consuming. With our current setup, each network has to be run for ca. $12 \mathrm{~h}$, and to 
Table 2

Artificial Neural Network configuration for the prediction of $\mathrm{SST}_{100}$

\begin{tabular}{|c|c|c|c|c|c|c|c|c|c|c|}
\hline & N1 & $\mathrm{N} 2$ & N3 & N4 & N5 & N6 & N7 & N8 & N9 & N10 \\
\hline RMSEP & 0.759 & 0.722 & 0.723 & 0.768 & 0.715 & 0.721 & 0.740 & 0.705 & 0.618 & 1.003 \\
\hline $\mathrm{r}$ & 0.959 & 0.967 & 0.967 & 0.957 & 0.958 & 0.951 & 0.958 & 0.964 & 0.973 & 0.917 \\
\hline \multicolumn{11}{|l|}{ Species selection } \\
\hline Actinomma leptoderma/boreale group & $\mathrm{X}$ & $\mathrm{X}$ & $\mathrm{X}$ & & $\mathrm{X}$ & & $\mathrm{X}$ & $\mathrm{X}$ & $\mathrm{X}$ & $\mathrm{X}$ \\
\hline Actinomma leptoderma longispina & $\mathrm{X}$ & & $\mathrm{X}$ & $\mathrm{X}$ & $\mathrm{X}$ & $\mathrm{X}$ & & $\mathrm{X}$ & $\mathrm{X}$ & $\mathrm{X}$ \\
\hline Actinomma medianum & & & & $\mathrm{X}$ & & & & $\mathrm{X}$ & & \\
\hline Actinomma popofskii & & $\mathrm{X}$ & $\mathrm{X}$ & & $\mathrm{X}$ & $\mathrm{X}$ & & & & \\
\hline Actinomma sp. 2 & $\mathrm{X}$ & & & & & & $\mathrm{X}$ & $\mathrm{X}$ & & \\
\hline Amphimelissa setosa & & $\mathrm{X}$ & & & $\mathrm{X}$ & $\mathrm{X}$ & & & & $\mathrm{X}$ \\
\hline Artobotrys boreale & & $\mathrm{X}$ & & $\mathrm{X}$ & & $\mathrm{X}$ & $\mathrm{X}$ & $\mathrm{X}$ & $\mathrm{X}$ & \\
\hline Artostrobus joergenseni & & & & $\mathrm{X}$ & $\mathrm{X}$ & $\mathrm{X}$ & & & $\mathrm{X}$ & \\
\hline Ceratocyrtis galeus & $\mathrm{X}$ & $\mathrm{X}$ & & & & & & & $\mathrm{X}$ & \\
\hline Ceratocyrtis histricosus & & & & & & $\mathrm{X}$ & & & & \\
\hline Corocalyptra craspedota & & & $\mathrm{X}$ & $\mathrm{X}$ & & $\mathrm{X}$ & & & & \\
\hline Larcospira minor & $\mathrm{X}$ & & $\mathrm{X}$ & $\mathrm{X}$ & $\mathrm{X}$ & $\mathrm{X}$ & $\mathrm{X}$ & $\mathrm{X}$ & $\mathrm{X}$ & $\mathrm{X}$ \\
\hline Larcospira sp. 1 & $\mathrm{X}$ & $\mathrm{X}$ & & $\mathrm{X}$ & & $\mathrm{X}$ & & $\mathrm{X}$ & $\mathrm{X}$ & $\mathrm{X}$ \\
\hline Lipmanella xiphephorum & $\mathrm{X}$ & & & $\mathrm{X}$ & & $\mathrm{X}$ & & & & \\
\hline Lithelius spiralis & $\mathrm{X}$ & & $\mathrm{X}$ & & & & $\mathrm{X}$ & $\mathrm{X}$ & & \\
\hline Lithomelissa hystrix & & & $\mathrm{X}$ & $\mathrm{X}$ & $\mathrm{X}$ & $\mathrm{X}$ & $\mathrm{X}$ & & $\mathrm{X}$ & \\
\hline Lithomelissa setosa & $\mathrm{X}$ & $\mathrm{X}$ & $\mathrm{X}$ & $\mathrm{X}$ & $\mathrm{X}$ & $\mathrm{X}$ & $\mathrm{X}$ & $\mathrm{X}$ & $\mathrm{X}$ & $\mathrm{X}$ \\
\hline Lithomelissa sp. aff. L. stigi & $\mathrm{X}$ & $\mathrm{X}$ & $\mathrm{X}$ & & & $\mathrm{X}$ & $\mathrm{X}$ & & & \\
\hline Lithomelissa thoracites & $\mathrm{X}$ & $\mathrm{X}$ & $\mathrm{X}$ & $\mathrm{X}$ & $\mathrm{X}$ & $\mathrm{X}$ & $\mathrm{X}$ & $\mathrm{X}$ & $\mathrm{X}$ & $\mathrm{X}$ \\
\hline Lithomitra lineata & $\mathrm{X}$ & $\mathrm{X}$ & $\mathrm{X}$ & $\mathrm{X}$ & $\mathrm{X}$ & & & & $\mathrm{X}$ & \\
\hline Phorticium clevei & $\mathrm{X}$ & $\mathrm{X}$ & $\mathrm{X}$ & & $\mathrm{X}$ & $\mathrm{X}$ & $\mathrm{X}$ & $\mathrm{X}$ & $\mathrm{X}$ & $\mathrm{X}$ \\
\hline Phorticium sp. 1 & & $\mathrm{X}$ & & & $\mathrm{X}$ & $\mathrm{X}$ & & $\mathrm{X}$ & $\mathrm{X}$ & \\
\hline Plagiacantha arachnoides & & $\mathrm{X}$ & $\mathrm{X}$ & $\mathrm{X}$ & $\mathrm{X}$ & $\mathrm{X}$ & $\mathrm{X}$ & & $\mathrm{X}$ & \\
\hline Plectacantha oikiskos & $\mathrm{X}$ & & $\mathrm{X}$ & $\mathrm{X}$ & & $\mathrm{X}$ & $\mathrm{X}$ & $\mathrm{X}$ & $\mathrm{X}$ & \\
\hline Pseudodictyophimus gracilipes & & $\mathrm{X}$ & & $\mathrm{X}$ & & $\mathrm{X}$ & $\mathrm{X}$ & $\mathrm{X}$ & $\mathrm{X}$ & \\
\hline Sethoconus tabulatus & & $\mathrm{X}$ & $\mathrm{X}$ & $\mathrm{X}$ & $\mathrm{X}$ & & & & $\mathrm{X}$ & $\mathrm{X}$ \\
\hline Streblacantha circumtexta & & $\mathrm{X}$ & $\mathrm{X}$ & $\mathrm{X}$ & & $\mathrm{X}$ & & & $\mathrm{X}$ & \\
\hline Streblacantha sp.1 & & & $\mathrm{X}$ & & & & $\mathrm{X}$ & & & \\
\hline Stylatractus sp. 1 & & $\mathrm{X}$ & $\mathrm{X}$ & $\mathrm{X}$ & & $\mathrm{X}$ & $\mathrm{X}$ & & $\mathrm{X}$ & $\mathrm{X}$ \\
\hline Tholospyris gephyristes & & & & $\mathrm{X}$ & $\mathrm{X}$ & $\mathrm{X}$ & & & $\mathrm{X}$ & \\
\hline
\end{tabular}

Root Mean Square Errors of Prediction (RMSEP), correlation coefficients (r) for the relationship between observed and predicted SST in the test sets, and the taxa used are reported for each network configuration.

be able to obtain an accurate estimate of the RMSEP, the same dataset needs to be run several times with different training/test set splits, selected by the program.

\subsection{Core sampling and chronology}

All radiolarian samples used in this study have been described in previous papers. See: Cortese et al. (2003), for the 161 surface sediment samples (shown in the inset of Fig. 1), and Dolven et al. (2002), for core HM79-4 $\left(63^{\circ} 06^{\prime} \mathrm{N}, 02^{\circ} 33^{\prime} \mathrm{E}\right)$ and core MD95-2011 $\left(66^{\circ} 58^{\prime} \mathrm{N}, 07^{\circ} 38^{\prime} \mathrm{E}\right)$. The studied cores were recovered by the R/V Håkon Mosby (during a campaign organized by the Geological Institute, University of Bergen), and by the $\mathrm{R} / \mathrm{V}$ Marion Dufresne (during IMAGES cruise 101), respectively. A total of 64 samples were collected from a $1.85 \mathrm{~m}$ $(55.5-240.4 \mathrm{~cm})$ long section of core HM79-4, spanning $9880-13,400{ }^{14} \mathrm{C}$ years B.P., with a resulting average sedimentation rate of $52 \mathrm{~cm} / 1000$ years, and an average sampling resolution of 55 years. Core MD95-2011 provided 126 samples from a 6.40 $\mathrm{m}$ long section $(15.5-655.5 \mathrm{~cm})$, dated at $600-9770$ ${ }^{14} \mathrm{C}$ years B.P., thus resulting in an average sedimentation rate of $70 \mathrm{~cm} / 1000$ years, and an average 
sampling resolution of 73 years. Note that the first few hundred years in core MD95-2011 were lost during coring operations.
The Vedde Ash layer (Birks et al., 1996), sixteen AMS ${ }^{14} \mathrm{C}$-datings of Neogloboquadrina pachyderma (Koc-Karpuz and Jansen, 1992), and the peak occur-

Plate I. Spumellarians. All figures, scale bar is $100 \mu \mathrm{m}$ (see page 317).

1. Actinomma boreale, St. V27-94, Norwegian Sea

2. Actinomma popofskii Petrushevskaya, 1968 (Actinomma sp.1 in Cortese et al., 2003), St. GIK17056, North Atlantic

3. Actinomma popofskii Petrushevskaya, 1968 (Actinomma sp.1 in Cortese et al., 2003), St. GIK17056, North Atlantic

4. Actinomma leptoderma longispina, St. GIK23417, North Atlantic

5. Actinomma sp. 2, St. 10, Nærøyfjorden

6. Actinomma medianum, St. GIK23417, North Atlantic

7. Larcospira minor, St. V27-94, Norwegian Sea

8. Larcospira minor, St. V27-94, Norwegian Sea

9. Actinomma leptoderma longispina, St. V27-94, Norwegian Sea

10. Stylatractus sp. 1, St. GIK17056, North Atlantic

11. Actinomma leptoderma, St. V27-94, Norwegian Sea

12. Lithelius spiralis, St. V27-94, Norwegian Sea

13. Phorticium (clevei) pylonium, St. V27-94, Norwegian Sea

14. Streblacantha circumtexta, St. 10, Nærøyfjorden

15. Phorticium sp. 1, St. GIK23413, North Atlantic

16. Larcospira sp. 1, St. V27-94, Norwegian Sea

17. Larcospira sp. 1, St. V27-94, Norwegian Sea

18. Streblacantha sp. 1, St. V27-94, Norwegian Sea

Plate II. Nassellarians. 1-8, 11 and 13, scale bar is $50 \mu \mathrm{m}$. All other figures, scale bar is $100 \mu \mathrm{m}$ (see page 318).

1. Amphimelissa setosa, St. V27-46, Norwegian Sea

2. Amphimelissa setosa, St. V27-94, Norwegian Sea

3. Artobotrys boreale, St. V27-94, Norwegian Sea

4. Artobotrys boreale, St. V27-94, Norwegian Sea

5. Artostrobus joergenseni, St. V27-94, Norwegian Sea

6. Artostrobus joergenseni, St. V27-46, Norwegian Sea

7. Lithomelissa thoracites, St. V27-94, Norwegian Sea

8. Lithomelissa setosa, St. V27-94, Norwegian Sea

9. Lithomitra lineata, St. V27-94, Norwegian Sea

10. Lithomitra lineata, St. V27-94, Norwegian Sea

11. Lithomelissa setosa, St. V27-94, Norwegian Sea

12. Lithomelissa sp. aff. L. stigi, St. V27-94, Norwegian Sea

13. Sethoconus tabulatus, St. V27-94, Norwegian Sea

14. Plectacantha oikiskos, St. V27-94, Norwegian Sea

15. Lithomelissa hystrix, St. V27-94, Norwegian Sea

16. Lithomelissa hystrix, St. V27-94, Norwegian Sea

17. Pseudodictyophimus gracilipes, St. V27-94, Norwegian Sea

18. Pseudodictyophimus gracilipes, St. V27-94, Norwegian Sea

19. Lithomelissa sp. aff. L. stigi, St. V27-94, Norwegian Sea

20. Pseudodictyophimus gracilipes, St. V27-94, Norwegian Sea

21. Ceratocyrtis galeus, St. V23-76, Norwegian Sea

22. Ceratocyrtis histricosus, St. V23-76, Norwegian Sea

23. Tholospyris gephyristes, St. V27-94, Norwegian Sea

24. Plagiacantha arachnoides, St. 25, Balsfjord

25. Ceratocyrtis galeus, St. V27-94, Norwegian Sea

26. Corocalyptra craspedota, St. V23-76, Norwegian Sea

27. Corocalyptra craspedota, St. V23-76, Norwegian Sea

28. Lipmanella xiphephorum, St. V23-76, Norwegian Sea 

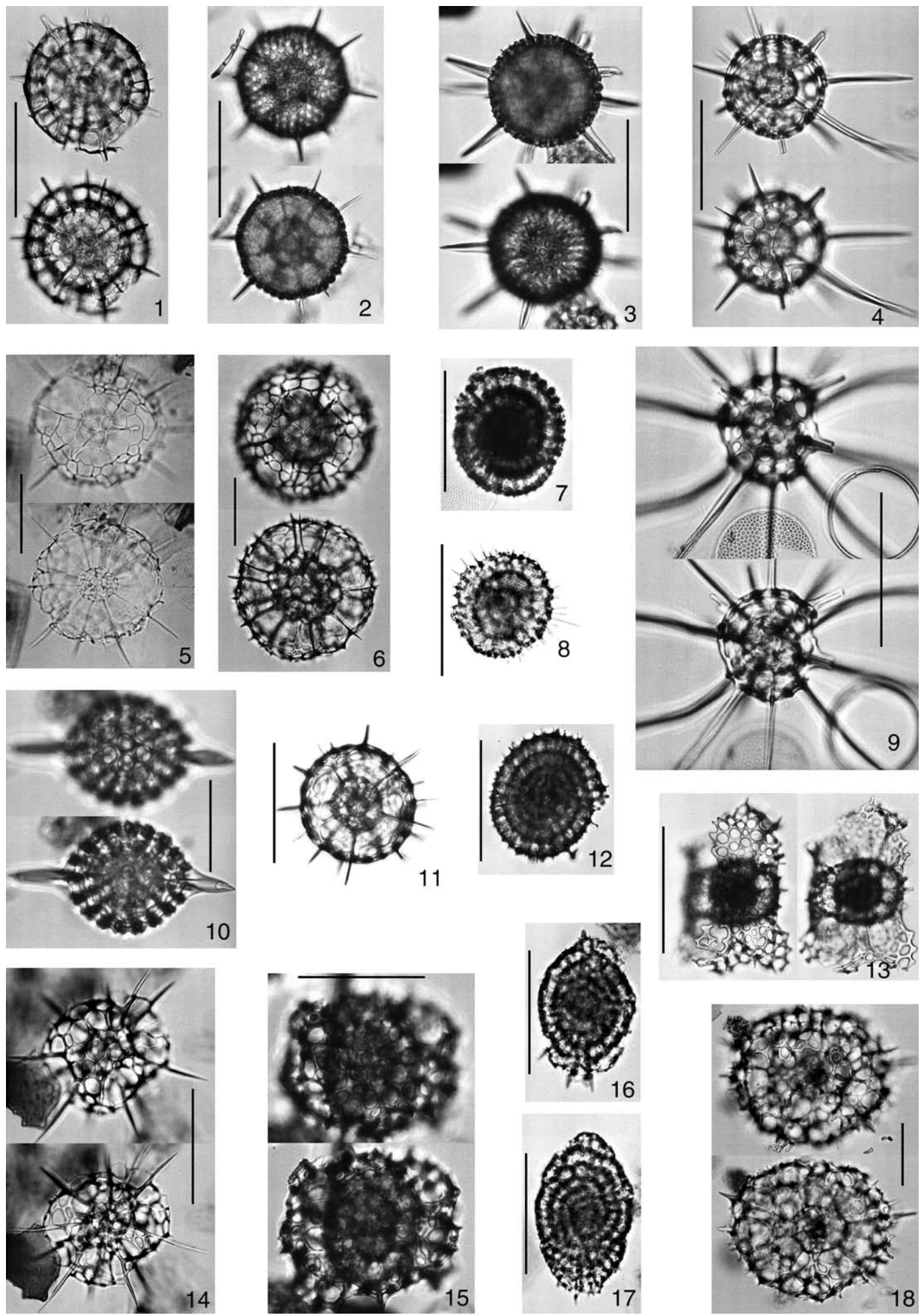

Plate I (caption on page 316). 

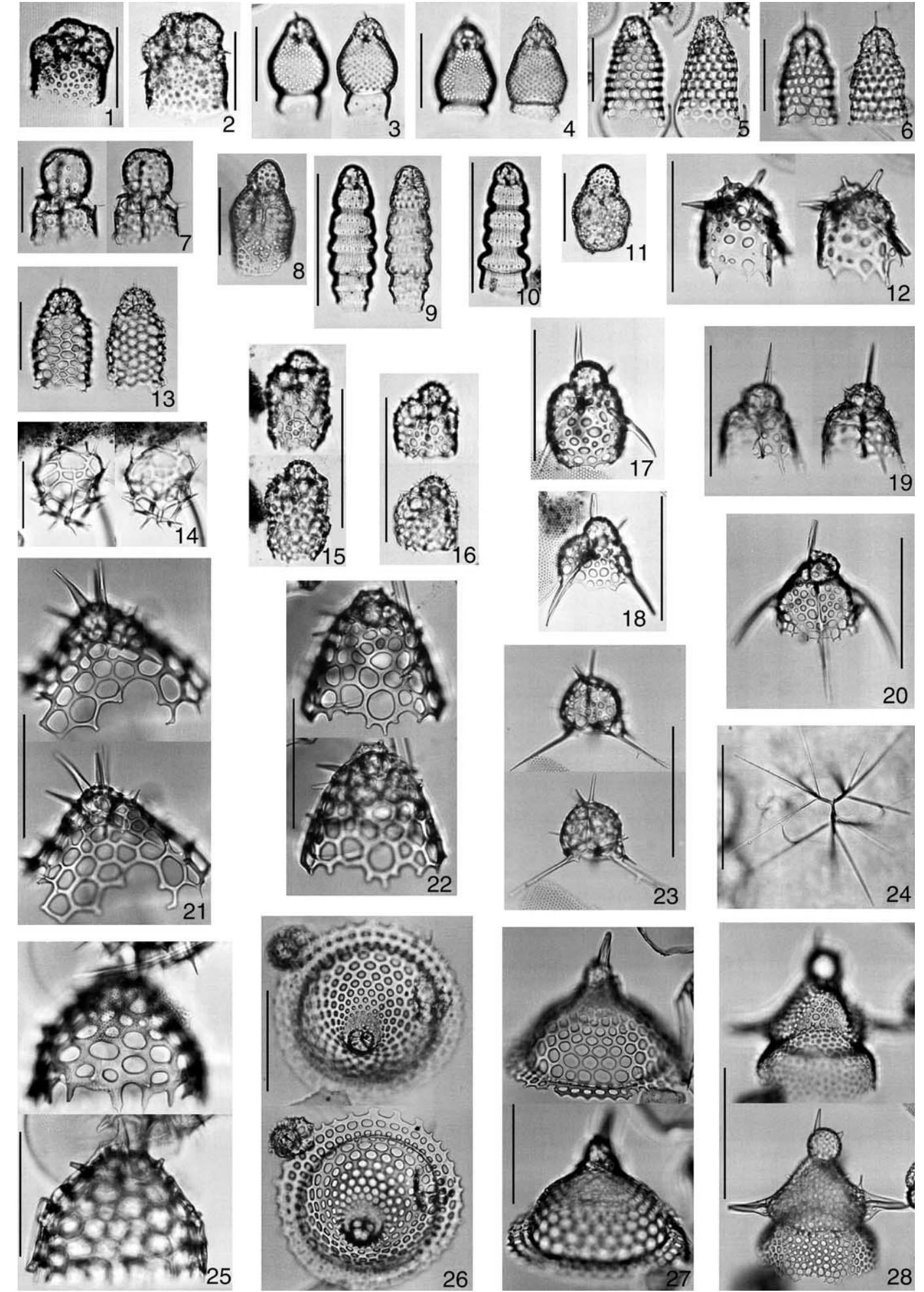

Plate II (caption on page 316).

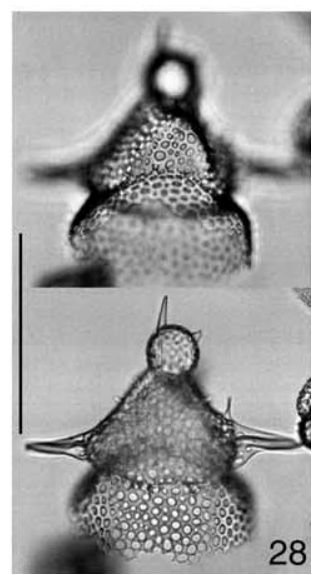


rence of the radiolarian species Rhizoplegma boreale (Dolven and Bjørklund, 2001) were used to develop the age model of the two cores and correlate them. The precision of the ${ }^{14} \mathrm{C}$ age determinations for the dating points is better than \pm 100 years for the Holocene section, and ca. \pm 150 years for the older part. All dating points, as well as the tie points for the synchronization of the calendar year chronology to the GISP ice core, are listed in Dolven et al. (2002). The correction of the previous age model, by peak-topeak correlation of our SST and the GISP $\delta^{18} \mathrm{O}$ records, was necessary to account for the assumingly longer residence time of seawater in the study area during glacial times, which tends to produce artificially older ages during these time intervals. In this paper we have used the calendar year chronology developed from correlation to the GISP ice core (Table 3) when plotting our figures. Further details on sampling, preparation and raw data production, as well as the chronology of the two cores, are available in Dolven et al. (2002) and Cortese et al. (2003).

\subsection{Hydrography}

We interpolated temperature data for the warm season (Summer Sea Temperature, SST) at the 161 surface sediment sample sites by using the multiplatform oceanography visualization software Ocean Data View (Schlitzer, 2000). This procedure was carried out automatically based on the geographic position of our cores and a regular overlay grid represented by the one degree-spaced World Ocean Atlas data (Conkright et al., 1998). SST represents seasonally averaged July-September (summer) temperatures for $100 \mathrm{~m}$ $\left(\mathrm{SST}_{100}\right)$ depth.

\subsection{Seasonality}

Regression techniques are often used to estimate both cold- and warm-season temperatures. We however reconstructed only warm-season temperatures, as we believe that the specimens embedded and preserved in the sediment are mainly those produced during spring/summer, when radiolarian peak production takes place. This is in good agreement with studies previously carried out in the Nordic Seas, based on sediment traps and on vertical profiles of both species and specimens abundances (Schröder-
Table 3

Downcore data for cores MD95-2011 and HM79-4: for each sample depth, the IKTF-SST 0 (Dolven et al., 2002) and the ANN-SST 100 and its $95 \%$ confidence interval are reported, along with the age assignment of each sample (in calendar years Before Present), for both the original age model (as shown in Dolven et al., 2002; Table 3 ), and for the GISP-synchronized age model used in the present paper (see text for details).

\begin{tabular}{|c|c|c|c|c|c|}
\hline $\begin{array}{l}\text { Depth } \\
\text { (cm) }\end{array}$ & $\begin{array}{l}\text { Calendar } \\
\text { age }\end{array}$ & $\begin{array}{l}\text { Age } \\
\text { GISP }\end{array}$ & $\begin{array}{l}\text { SST0 } \\
\text { IKTF }\end{array}$ & $\begin{array}{l}\text { SST100 } \\
\text { ANN }\end{array}$ & $\begin{array}{l}\text { SST100 } \\
\text { ANN }\end{array}$ \\
\hline MD95-2011 & (years BP) & (years BP) & $\left({ }^{\circ} \mathrm{C}\right)$ & $\left({ }^{\circ} \mathrm{C}\right)$ & $95 \% \mathrm{CI}\left({ }^{\circ} \mathrm{C}\right)$ \\
\hline 15.5 & 567 & 1001 & 11.8 & 7.0 & 0.2 \\
\hline 19.5 & 581 & 1013 & 12.8 & 6.7 & 0.4 \\
\hline 24.5 & 600 & 1030 & 12.5 & 6.3 & 0.5 \\
\hline 29.5 & 618 & 1046 & 12.8 & 6.5 & 0.3 \\
\hline 35.5 & 641 & 1066 & 12.7 & 6.7 & 0.3 \\
\hline 41.5 & 663 & 1085 & 12.3 & 6.9 & 0.2 \\
\hline 44.5 & 674 & 1095 & 12.6 & 6.9 & 0.2 \\
\hline 49.5 & 711 & 1127 & 11.1 & 5.7 & 0.7 \\
\hline 55.5 & 788 & 1184 & 13.0 & 6.3 & 0.3 \\
\hline 59.5 & 839 & 1221 & 12.7 & 6.8 & 0.2 \\
\hline 65.5 & 917 & 1279 & 12.1 & 4.9 & 0.4 \\
\hline 69.5 & 968 & 1316 & 12.0 & 6.1 & 0.5 \\
\hline 75.5 & 1026 & 1359 & 11.7 & 6.1 & 0.4 \\
\hline 81.5 & 1080 & 1399 & 11.6 & 5.7 & 0.4 \\
\hline 85.5 & 1116 & 1426 & 10.9 & 4.3 & 0.7 \\
\hline 90.5 & 1164 & 1461 & 11.9 & 6.9 & 0.2 \\
\hline 95.5 & 1225 & 1506 & 11.1 & 4.6 & 0.3 \\
\hline 101.5 & 1298 & 1560 & 11.4 & 4.0 & 0.4 \\
\hline 105.5 & 1347 & 1596 & 12.1 & 6.6 & 0.4 \\
\hline 109.5 & 1395 & 1631 & 12.0 & 6.0 & 0.4 \\
\hline 115.5 & 1468 & 1685 & 11.8 & 5.2 & 0.6 \\
\hline 124.5 & 1578 & 1766 & 11.8 & 6.0 & 0.7 \\
\hline 129.5 & 1639 & 1811 & 10.5 & 5.3 & 0.4 \\
\hline 135.5 & 1712 & 1897 & 11.0 & 4.8 & 0.4 \\
\hline 139.5 & 1761 & 1954 & 12.0 & 6.4 & 0.5 \\
\hline 145.5 & 1834 & 2039 & 12.4 & 6.9 & 0.2 \\
\hline 148.5 & 1870 & 2082 & 12.6 & 6.8 & 0.3 \\
\hline 155.5 & 1971 & 2200 & 11.3 & 5.1 & 0.6 \\
\hline 160.5 & 2082 & 2330 & 12.0 & 6.6 & 0.2 \\
\hline 165.5 & 2194 & 2461 & 12.1 & 6.2 & 0.3 \\
\hline 170.5 & 2306 & 2592 & 12.0 & 6.6 & 0.2 \\
\hline 175.5 & 2379 & 2678 & 12.3 & 6.2 & 0.4 \\
\hline 180.5 & 2452 & 2763 & 12.4 & 6.4 & 0.4 \\
\hline 185.5 & 2526 & 2850 & 12.8 & 6.6 & 0.3 \\
\hline 190.5 & 2599 & 2936 & 12.5 & 6.5 & 0.3 \\
\hline 195.5 & 2672 & 3021 & 11.8 & 4.8 & 0.5 \\
\hline 200.5 & 2745 & 3107 & 12.1 & 6.3 & 0.3 \\
\hline 205.5 & 2818 & 3192 & 12.1 & 6.0 & 0.4 \\
\hline 210.5 & 2891 & 3278 & 12.1 & 5.0 & 0.4 \\
\hline 215.5 & 2965 & 3364 & 12.4 & 6.3 & 0.3 \\
\hline 219.5 & 3023 & 3432 & 12.5 & 6.5 & 0.4 \\
\hline 225.5 & 3111 & 3536 & 11.6 & 4.7 & 0.6 \\
\hline 230.5 & 3184 & 3621 & 11.6 & 5.1 & 0.7 \\
\hline 235.5 & 3257 & 3707 & 12.4 & 6.6 & 0.3 \\
\hline
\end{tabular}

(continued on next page) 
Table 3 (continued)

\begin{tabular}{|c|c|c|c|c|c|}
\hline $\begin{array}{l}\text { Depth } \\
(\mathrm{cm})\end{array}$ & $\begin{array}{l}\text { Calendar } \\
\text { age }\end{array}$ & $\begin{array}{l}\text { Age } \\
\text { GISP }\end{array}$ & $\begin{array}{l}\text { SST0 } \\
\text { IKTF } \\
\end{array}$ & $\begin{array}{l}\text { SST100 } \\
\text { ANN }\end{array}$ & $\begin{array}{l}\text { SST100 } \\
\text { ANN }\end{array}$ \\
\hline MD95-2011 & (years BP) & (years BP) & $\left({ }^{\circ} \mathrm{C}\right)$ & $\left({ }^{\circ} \mathrm{C}\right)$ & $95 \% \mathrm{CI}\left({ }^{\circ} \mathrm{C}\right)$ \\
\hline 240.5 & 3331 & 3793 & 12.6 & 6.5 & 0.4 \\
\hline 245.5 & 3404 & 3879 & 11.6 & 4.7 & 0.4 \\
\hline 250.5 & 3477 & 3964 & 11.5 & 5.3 & 0.4 \\
\hline 255.5 & 3550 & 4050 & 12.9 & 6.6 & 0.4 \\
\hline 260.5 & 3623 & 4135 & 11.3 & 4.2 & 0.5 \\
\hline 265.5 & 3696 & 4221 & 11.1 & 3.8 & 0.6 \\
\hline 270.5 & 3768 & 4305 & 11.3 & 4.9 & 0.5 \\
\hline 275.5 & 3834 & 4383 & 11.4 & 4.6 & 0.4 \\
\hline 280.5 & 3900 & 4460 & 12.1 & 6.0 & 0.3 \\
\hline 285.5 & 3966 & 4537 & 12.5 & 6.2 & 0.4 \\
\hline 289.5 & 4019 & 4599 & 11.5 & 4.4 & 0.4 \\
\hline 295.5 & 4099 & 4693 & 10.9 & 4.3 & 0.4 \\
\hline 298.5 & 4138 & 4729 & 11.9 & 6.0 & 0.5 \\
\hline 306.5 & 4244 & 4826 & 12.8 & 5.4 & 0.5 \\
\hline 312.5 & 4323 & 4899 & 11.8 & 5.2 & 0.4 \\
\hline 326.5 & 4527 & 5086 & 12.3 & 6.5 & 0.3 \\
\hline 329.5 & 4576 & 5131 & 11.8 & 6.2 & 0.5 \\
\hline 334.5 & 4657 & 5205 & 11.7 & 5.8 & 0.4 \\
\hline 339.5 & 4739 & 5280 & 11.2 & 5.4 & 0.4 \\
\hline 344.5 & 4820 & 5355 & 12.0 & 5.6 & 0.4 \\
\hline 349.5 & 4902 & 5430 & 11.7 & 5.0 & 0.3 \\
\hline 354.5 & 4984 & 5505 & 12.1 & 5.8 & 0.3 \\
\hline 359.5 & 5065 & 5580 & 12.5 & 5.8 & 0.4 \\
\hline 364.5 & 5147 & 5655 & 12.3 & 6.6 & 0.3 \\
\hline 369.5 & 5228 & 5729 & 11.8 & 5.7 & 0.5 \\
\hline 374.5 & 5310 & 5804 & 11.3 & 5.1 & 0.6 \\
\hline 378.5 & 5375 & 5864 & 12.0 & 6.6 & 0.3 \\
\hline 384.5 & 5473 & 5954 & 11.5 & 6.5 & 0.3 \\
\hline 389.5 & 5554 & 6028 & 11.5 & 5.4 & 0.5 \\
\hline 394.5 & 5636 & 6104 & 11.5 & 5.2 & 0.3 \\
\hline 399.5 & 5717 & 6178 & 12.1 & 5.6 & 0.5 \\
\hline 404.5 & 5799 & 6253 & 12.5 & 6.8 & 0.2 \\
\hline 409.5 & 5881 & 6329 & 11.7 & 5.9 & 0.4 \\
\hline 414.5 & 5962 & 6403 & 12.1 & 6.3 & 0.5 \\
\hline 419.5 & 6044 & 6478 & 12.4 & 6.7 & 0.3 \\
\hline 424.5 & 6125 & 6553 & 11.6 & 5.4 & 0.3 \\
\hline 428.5 & 6190 & 6612 & 11.7 & 6.3 & 0.3 \\
\hline 434.5 & 6288 & 6702 & 12.0 & 6.5 & 0.3 \\
\hline 438.5 & 6354 & 6763 & 12.3 & 6.5 & 0.2 \\
\hline 444.5 & 6451 & 6852 & 11.5 & 5.3 & 0.8 \\
\hline 448.5 & 6517 & 6912 & 12.0 & 5.6 & 0.3 \\
\hline 455.5 & 6631 & 7017 & 11.8 & 6.5 & 0.3 \\
\hline 460.5 & 6712 & 7091 & 11.3 & 5.5 & 0.6 \\
\hline 465.5 & 6794 & 7167 & 12.3 & 7.2 & 0.1 \\
\hline 470.5 & 6876 & 7242 & 12.0 & 6.9 & 0.2 \\
\hline 475.5 & 6957 & 7316 & 11.9 & 6.9 & 0.3 \\
\hline 480.5 & 7039 & 7391 & 11.5 & 5.7 & 0.4 \\
\hline 485.5 & 7120 & 7466 & 11.1 & 6.6 & 0.3 \\
\hline 490.5 & 7202 & 7541 & 10.4 & 4.7 & 0.8 \\
\hline 495.5 & 7283 & 7618 & 12.0 & 7.3 & 0.2 \\
\hline 500.5 & 7365 & 7696 & 10.7 & 4.7 & 0.7 \\
\hline 505.5 & 7446 & 7774 & 11.9 & 6.7 & 0.4 \\
\hline
\end{tabular}

Table 3 (continued)

\begin{tabular}{|c|c|c|c|c|c|}
\hline $\begin{array}{l}\text { Depth } \\
(\mathrm{cm})\end{array}$ & $\begin{array}{l}\text { Calendar } \\
\text { age }\end{array}$ & $\begin{array}{l}\text { Age } \\
\text { GISP }\end{array}$ & $\begin{array}{l}\text { SST0 } \\
\text { IKTF } \\
\end{array}$ & $\begin{array}{l}\text { SST100 } \\
\text { ANN } \\
\end{array}$ & $\begin{array}{l}\text { SST100 } \\
\text { ANN }\end{array}$ \\
\hline MD95-2011 & (years BP) & (years BP) & $\left({ }^{\circ} \mathrm{C}\right)$ & $\left({ }^{\circ} \mathrm{C}\right)$ & $95 \% \mathrm{CI}\left({ }^{\circ} \mathrm{C}\right)$ \\
\hline 510.5 & 7528 & 7852 & 12.2 & 6.9 & 0.2 \\
\hline 515.5 & 7609 & 7929 & 10.7 & 4.7 & 0.5 \\
\hline 520.5 & 7691 & 8007 & 10.7 & 5.6 & 0.4 \\
\hline 525.5 & 7819 & 8129 & 10.5 & 4.9 & 0.6 \\
\hline 530.5 & 7948 & 8252 & 10.4 & 5.3 & 0.6 \\
\hline 535.5 & 8076 & 8400 & 12.5 & 7.4 & 0.2 \\
\hline 540.5 & 8204 & 8548 & 11.9 & 7.6 & 0.2 \\
\hline 545.5 & 8332 & 8697 & 11.6 & 6.9 & 0.2 \\
\hline 550.5 & 8461 & 8846 & 13.0 & 7.6 & 0.2 \\
\hline 555.5 & 8589 & 8994 & 12.4 & 7.2 & 0.2 \\
\hline 560.5 & 8717 & 9142 & 12.0 & 7.1 & 0.2 \\
\hline 565.5 & 8846 & 9292 & 12.1 & 7.6 & 0.2 \\
\hline 570.5 & 8974 & 9440 & 12.6 & 7.2 & 0.1 \\
\hline 575.5 & 9102 & 9588 & 12.0 & 7.4 & 0.1 \\
\hline 580.5 & 9231 & 9737 & 12.2 & 7.2 & 0.1 \\
\hline 585.5 & 9359 & 9886 & 12.1 & 7.2 & 0.2 \\
\hline 590.5 & 9487 & 10,034 & 11.7 & 7.3 & 0.2 \\
\hline 595.5 & 9615 & 10,182 & 12.4 & 7.5 & 0.2 \\
\hline 602.5 & 9795 & 10,390 & 12.0 & 7.4 & 0.2 \\
\hline 605.5 & 9872 & 10,480 & 11.4 & 6.6 & 0.3 \\
\hline 610.5 & 10,000 & 10,628 & 12.2 & 7.4 & 0.1 \\
\hline 615.5 & 10,128 & 10,776 & 11.9 & 7.3 & 0.2 \\
\hline 620.5 & 10,256 & 10,924 & 12.3 & 7.8 & 0.2 \\
\hline 625.5 & 10,385 & 11,074 & 12.5 & 7.9 & 0.3 \\
\hline 630.5 & 10,513 & 11,222 & 11.9 & 7.2 & 0.2 \\
\hline 635.5 & 10,641 & 11,370 & 10.0 & 6.2 & 0.5 \\
\hline 640.5 & 10,770 & 11,407 & 11.0 & 7.3 & 0.1 \\
\hline 645.5 & 10,898 & 11,444 & 10.7 & 6.8 & 0.3 \\
\hline 650.5 & 11,026 & 11,480 & 12.0 & 7.7 & 0.2 \\
\hline 655.5 & 11,155 & 11,517 & 11.4 & 6.8 & 0.3 \\
\hline $\begin{array}{l}\text { Depth } \\
(\mathrm{cm})\end{array}$ & $\begin{array}{l}\text { Calendar } \\
\text { age }\end{array}$ & $\begin{array}{l}\text { Age } \\
\text { GISP } \\
\end{array}$ & $\begin{array}{l}\text { SST0 } \\
\text { IKTF } \\
\end{array}$ & $\begin{array}{l}\text { SST100 } \\
\text { ANN } \\
\end{array}$ & $\begin{array}{l}\text { SST100 } \\
\text { ANN }\end{array}$ \\
\hline HM79-4 & (years BP) & (years BP) & $\left({ }^{\circ} \mathrm{C}\right)$ & $\left({ }^{\circ} \mathrm{C}\right)$ & $95 \% \mathrm{CI}\left({ }^{\circ} \mathrm{C}\right)$ \\
\hline 55.5 & 11,155 & 11,517 & 10.5 & 7.5 & 0.2 \\
\hline 60.5 & 11,275 & 11,552 & 9.9 & 7.0 & 0.2 \\
\hline 65.5 & 11,394 & 11,586 & 8.9 & 3.1 & 0.5 \\
\hline 70.5 & 11,514 & 11,620 & 7.4 & 1.3 & 0.7 \\
\hline 75.5 & 11,633 & 11,655 & 7.2 & 0.7 & 0.3 \\
\hline 80.5 & 11,753 & 11,689 & 7.1 & 0.8 & 0.2 \\
\hline 84.5 & 11,849 & 11,777 & 7.3 & 0.9 & 0.6 \\
\hline 86.5 & 11,896 & 11,821 & 7.6 & 1.5 & 0.6 \\
\hline 91.5 & 12,066 & 11,977 & 7.5 & 1.6 & 0.6 \\
\hline 95.5 & 12,297 & 12,190 & 7.6 & 1.1 & 0.7 \\
\hline 100.5 & 12,584 & 12,455 & 8.0 & 1.2 & 0.8 \\
\hline 103.0 & 12,728 & 12,588 & 7.3 & 0.8 & 0.3 \\
\hline 105.5 & 12,872 & 12,720 & 7.5 & 0.8 & 0.6 \\
\hline 108.0 & 13,016 & 12,853 & 7.4 & 0.8 & 0.6 \\
\hline 110.5 & 13,146 & 12,900 & 8.2 & 1.8 & 0.5 \\
\hline 113.0 & 13,218 & 12,926 & 8.6 & 1.6 & 0.6 \\
\hline
\end{tabular}


Table 3 (continued)

\begin{tabular}{|c|c|c|c|c|c|}
\hline $\begin{array}{l}\text { Depth } \\
(\mathrm{cm})\end{array}$ & $\begin{array}{l}\text { Calendar } \\
\text { age }\end{array}$ & $\begin{array}{l}\text { Age } \\
\text { GISP }\end{array}$ & $\begin{array}{l}\text { SST0 } \\
\text { IKTF } \\
\end{array}$ & $\begin{array}{l}\text { SST100 } \\
\text { ANN }\end{array}$ & $\begin{array}{l}\text { SST100 } \\
\text { ANN }\end{array}$ \\
\hline HM79-4 & (years BP) & (years BP) & $\left({ }^{\circ} \mathrm{C}\right)$ & $\left({ }^{\circ} \mathrm{C}\right)$ & $95 \% \mathrm{CI}\left({ }^{\circ} \mathrm{C}\right)$ \\
\hline 115.5 & 13,290 & 12,951 & 8.4 & 1.4 & 0.6 \\
\hline 118.0 & 13,363 & 12,978 & 8.3 & 1.2 & 0.7 \\
\hline 120.5 & 13,435 & 13,004 & 7.6 & 1.1 & 0.6 \\
\hline 123.5 & 13,522 & 13,035 & 7.6 & 0.8 & 0.6 \\
\hline 127.5 & 13,638 & 13,076 & 7.8 & 2.0 & 0.6 \\
\hline 130.5 & 13,724 & 13,107 & 7.6 & 0.7 & 0.4 \\
\hline 134.0 & 13,826 & 13,144 & 7.4 & 0.7 & 0.5 \\
\hline 137.0 & 13,913 & 13,215 & 8.3 & 1.5 & 0.7 \\
\hline 140.5 & 14,014 & 13,297 & 8.5 & 1.2 & 0.7 \\
\hline 143.0 & 14,086 & 13,356 & 7.9 & 1.1 & 0.6 \\
\hline 146.5 & 14,151 & 13,409 & 8.5 & 1.5 & 0.7 \\
\hline 150.0 & 14,167 & 13,422 & 9.6 & 2.4 & 0.8 \\
\hline 153.0 & 14,180 & 13,432 & 8.3 & 1.1 & 0.7 \\
\hline 155.5 & 14,192 & 13,442 & 9.4 & 3.4 & 0.6 \\
\hline 158.0 & 14,203 & 13,451 & 9.5 & 3.4 & 0.6 \\
\hline 160.5 & 14,214 & 13,460 & 8.6 & 1.3 & 0.6 \\
\hline 163.0 & 14,226 & 13,470 & 8.5 & 1.7 & 0.6 \\
\hline 165.5 & 14,237 & 13,479 & 9.4 & 3.0 & 0.5 \\
\hline 168.0 & 14,248 & 13,488 & 9.3 & 3.4 & 0.7 \\
\hline 170.5 & 14,260 & 13,497 & 8.5 & 2.1 & 0.7 \\
\hline 173.0 & 14,271 & 13,506 & 8.0 & 0.9 & 0.4 \\
\hline 176.5 & 14,319 & 13,545 & 8.2 & 1.8 & 0.7 \\
\hline 178.5 & 14,371 & 13,588 & 8.9 & 2.2 & 0.6 \\
\hline 180.5 & 14,422 & 13,629 & 9.2 & 2.6 & 0.6 \\
\hline 183.0 & 14,487 & 13,682 & 8.2 & 0.6 & 0.2 \\
\hline 186.5 & 14,577 & 13,755 & 8.9 & 2.9 & 0.6 \\
\hline 188.5 & 14,629 & 13,798 & 8.8 & 2.2 & 0.6 \\
\hline 191.5 & 14,707 & 13,861 & 8.9 & 2.7 & 0.5 \\
\hline 193.0 & 14,745 & 13,892 & 9.3 & 3.2 & 1.1 \\
\hline 195.5 & 14,810 & 13,945 & 9.0 & 2.5 & 0.6 \\
\hline 198.0 & 14,875 & 13,998 & 9.1 & 4.7 & 0.5 \\
\hline 200.5 & 14,939 & 14,050 & 8.7 & 3.5 & 0.6 \\
\hline 203.0 & 15,004 & 14,087 & 9.9 & 6.2 & 0.4 \\
\hline 205.5 & 15,069 & 14,124 & 9.6 & 6.8 & 0.5 \\
\hline 208.0 & 15,133 & 14,161 & 9.6 & 6.5 & 0.7 \\
\hline 210.5 & 15,198 & 14,198 & 10.3 & 7.0 & 0.4 \\
\hline 213.0 & 15,263 & 14,235 & 10.1 & 7.2 & 0.2 \\
\hline 215.5 & 15,327 & 14,271 & 10.1 & 6.3 & 0.6 \\
\hline 218.0 & 15,392 & 14,308 & 10.3 & 6.5 & 0.5 \\
\hline 220.5 & 15,456 & 14,345 & 11.1 & 7.6 & 0.1 \\
\hline 223.0 & 15,521 & 14,382 & 11.0 & 7.0 & 0.2 \\
\hline 226.5 & 15,612 & 14,434 & 11.5 & 7.6 & 0.2 \\
\hline 228.0 & 15,650 & 14,455 & 11.6 & 7.2 & 0.2 \\
\hline 231.5 & 15,743 & 14,508 & 11.6 & 5.8 & 0.6 \\
\hline 233.0 & 15,783 & 14,531 & 12.1 & 6.7 & 0.4 \\
\hline 236.5 & 15,878 & 14,585 & 11.3 & 4.0 & 0.6 \\
\hline 238.5 & 15,932 & 14,616 & 11.4 & 4.6 & 0.7 \\
\hline 240.5 & 15,986 & 14,647 & 11.6 & 4.1 & 0.4 \\
\hline
\end{tabular}

Ritzrau, 1995). This is also supported by plankton studies in Korsfjorden, where the standing stock of living radiolarians reached highest values (individuals $/ \mathrm{m}^{3}$ ) during the summer (Bjørklund, 1974).

\section{Results}

\subsection{Network calibration phase}

Correlation coefficients between observed and predicted values are high $(r>0.95$, except for one partition; Table 2) and RMSEPs are satisfactorily low (0.62-1.00), meaning that down-core SSTs can be predicted within relatively low error limits. A graph showing the relationships between the observed and predicted $\mathrm{SST}_{100}$ in ten $\mathrm{ANN}$ runs is presented in Fig. 2, and summarized in Table 2. The main result of the network calibration phase is that the RMSEP estimates for $\mathrm{SST}_{100}$ are lower than for $\mathrm{SST}_{10}$ (Table 1). This confirms previous observations (Bjørklund et al., 1998), based on regressions only, on how the correlation between radiolarianderived factor components and SST increased with depth and was optimal at about $100 \mathrm{~m}$.

The reliability of our SST estimates is also confirmed by the match between the value obtained by ANN for the topmost core sample $(15.5 \mathrm{~cm}$, dated at 1001 years B.P.), and the observed World Ocean Atlas July-September SST (Conkright et al., 1998) at the MD95-2011 core location, i.e. the "expected" surface sediment value. The measured $\mathrm{SST}_{100}$ is $7.4{ }^{\circ} \mathrm{C}$, which matches well the ANNs sediment sample $\mathrm{SST}_{100}$ estimate $\left(7.1^{\circ} \mathrm{C}\right)$.

\subsection{Downcore $S S T_{100}$ predictions}

The estimated mean $\mathrm{SST}_{100}$ and 95\% confidence intervals over ten ANN runs are shown against age in Fig. 3. The results from ANN and ancillary information (depth, age, IKTF- and ANN-derived SSTs for each sample from cores HM79-4 and MD95-2011) are shown in Table 3. As the pre-Holocene evolution of our core is, in comparison to what is observed for the Holocene (see Discussion), quite typical, we will only describe it here and not discuss it in detail in the Next section, where we will instead focus on the Holocene portion. 

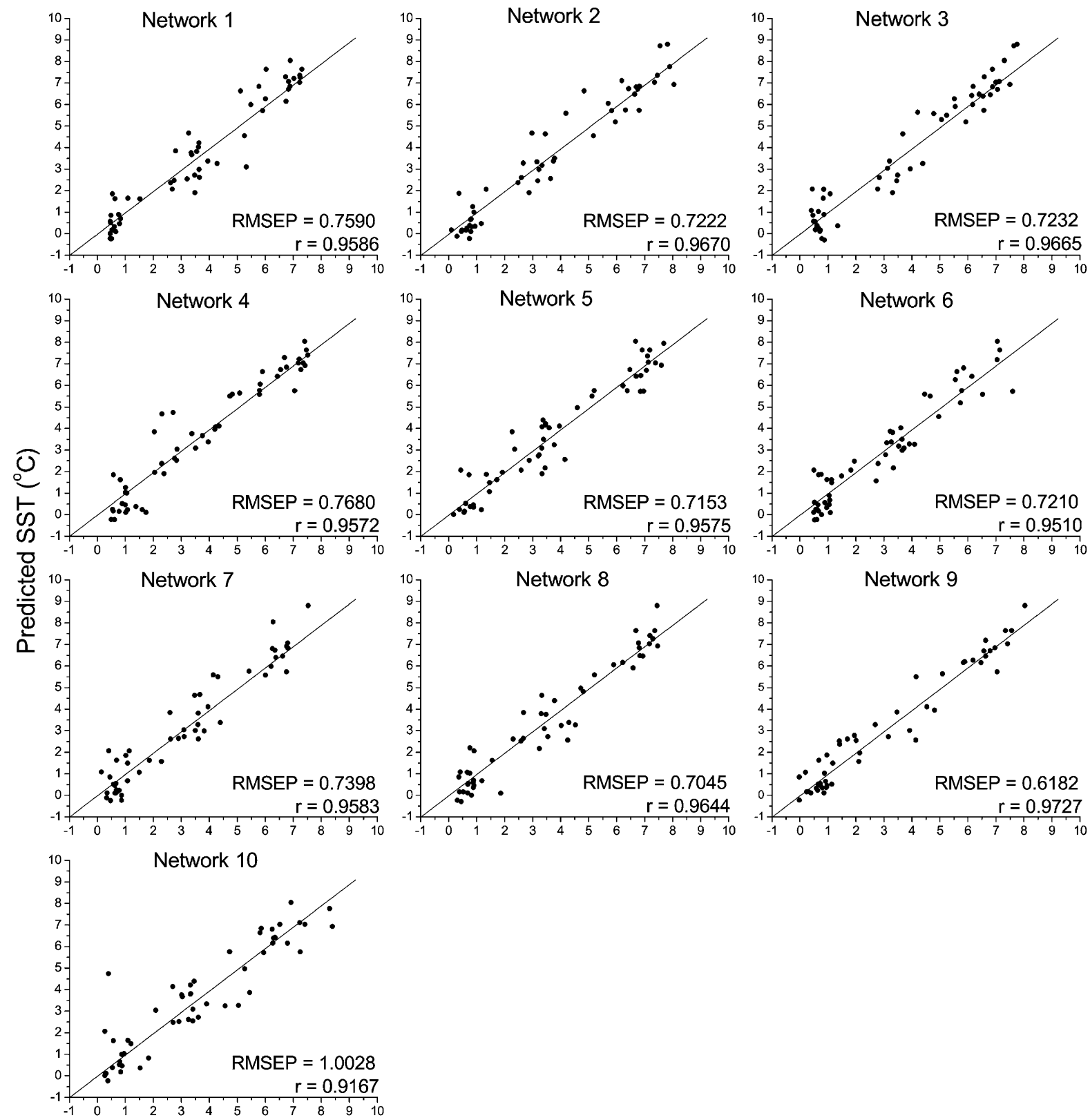

\section{Observed SST $\left({ }^{\circ} \mathrm{C}\right)$}

Fig. 2. Predicted vs. observed Summer Sea Temperatures (SST) for ten network runs (NGO software, $100 \mathrm{~m}$ water depth). RMSEPs and correlation coefficients are also reported (see also Table 2). The vertical bars represent the $95 \%$ confidence intervals based on the ten ANN runs.

The ANN paleo-SST estimates display, between 15 and $14 \mathrm{ka}$ (Fig. 3), during the first half of the Bølling/ Allerød, an interval with very high (over $7.5^{\circ} \mathrm{C}$ ) $\mathrm{SST}_{100}$. Along our record, values this high are other- wise only found during the early Holocene, an interval we named the Radiolarian Holocene Optimum Temperature Interval (RHOTI). The $\mathrm{SST}_{100}$ then abruptly drops to ca. $0.5{ }^{\circ} \mathrm{C}$ at $13.7 \mathrm{ka}$, with a $6.2{ }^{\circ} \mathrm{C}$ cooling 


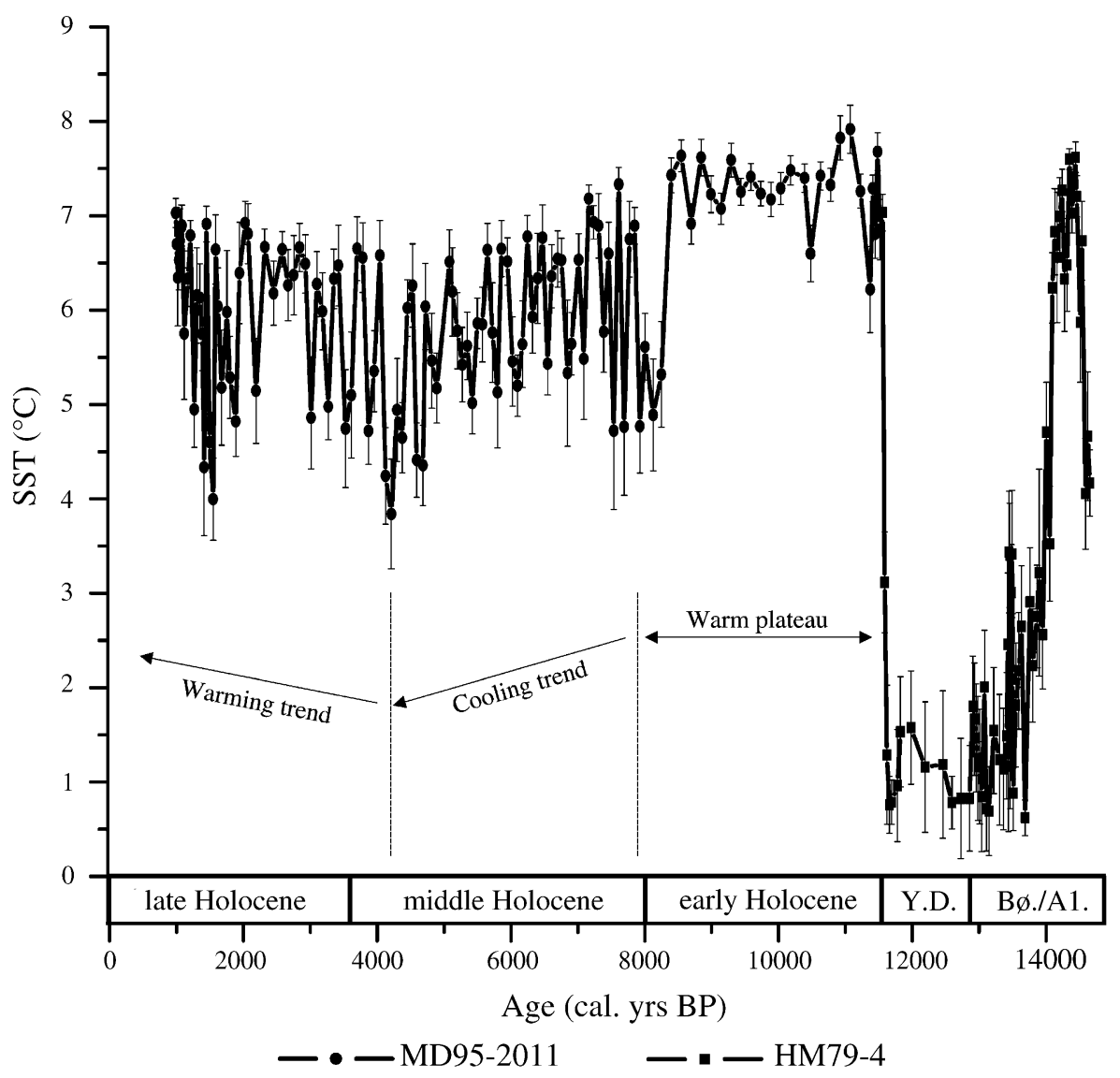

Fig. 3. SST predictions (average of ten runs) and standard deviations for cores MD95-2011 and HM79-4 (based on NGO software, $100 \mathrm{~m}$ water depth and setup "subset") are shown on an age scale, in calendar years Before Present. The Younger Dryas (Y.D.) and Bølling/Allerød (Bø./A1.) chronozones are after Mangerud et al. (1974). Vertical bars represent 95\% confidence intervals for the ten ANN-based SST estimates. The early Holocene plateau, the middle Holocene cooling trend, and the late Holocene warming trend are also schematically indicated.

taking place in 440 years. During the time interval including the second half of the Bølling/Allerød and the Younger Dryas (13.7-11.6 ka), temperatures remain stable at low values, except for several (4-5) short-lived, centennial, warming episodes, during which temperatures rise by ca. $1{ }^{\circ} \mathrm{C}$ over the background level. The transition to the Holocene takes place over 140 years, and rapidly brings $\mathrm{SST}_{100}$ to early Holocene levels, i.e. around $7.5^{\circ} \mathrm{C}$. This corresponds to an average warming rate of $4.9{ }^{\circ} \mathrm{C}$ per century $\left(6.8{ }^{\circ} \mathrm{C}\right.$ warming over only 140 years $)$, with rates up to more than twice as high between two consecutive samples (e.g. $11.5^{\circ} \mathrm{C}$ per century between the samples dated as 11,586 and 11,552 years BP, Table 3). This local oceanic warming rate is about half the maximum rate of atmospheric temperature change during the warming at the end of the Younger Dryas, which is reported to have occurred at rates as large as $10{ }^{\circ} \mathrm{C} / 50$ years for a significant part of the Northern Hemisphere (IPCC, 2001). In comparison to recent atmospheric warming rates, however, the observed local average oceanic rate of $4.9^{\circ} \mathrm{C}$ per century is quite high. In fact, model prognoses for the future, global mean, near-surface atmospheric temperature vary between 1 and $6{ }^{\circ} \mathrm{C}$ per century, with the current rate being lower than this range, at $0.1-0.2{ }^{\circ} \mathrm{C} /$ decade (IPCC, 2001).

The early Holocene (until ca. $8 \mathrm{ka}$ ) is characterized by relatively stable, warm conditions, with $\mathrm{SST}_{100}$ usually between 7 and $7.5^{\circ} \mathrm{C}$. A very rapid, almost 
$3{ }^{\circ} \mathrm{C}$ cooling takes place between 8.4 and $8.1 \mathrm{ka}$, and introduces an interval displaying a new style of climatic variability. In fact, after the recovery of $\mathrm{SST}_{100}$ to early Holocene levels at ca. $7.5 \mathrm{ka}$, the middle Holocene shows a clear trend of decreasing temperature, down to ca. $3.5{ }^{\circ} \mathrm{C}$ at $4.2 \mathrm{ka}$, with a superimposed centennial-scale variability, represented by repeated warmings and coolings of ca. $2{ }^{\circ} \mathrm{C}$ amplitude. A slightly stronger, ca. $3{ }^{\circ} \mathrm{C}$, warming starting at $4.2 \mathrm{ka}$ slightly predates the transition into the late Holocene (last $3.7 \mathrm{ka}$ ). Although $\mathrm{SST}_{100}$ still displays strong centennial variability during this interval, it follows a warming trend, with temperatures back to ca. $7{ }^{\circ} \mathrm{C}$ at 1100 years ago. The Kendall test indicates that this warming is statistically significant at the $5 \%$ level (Kendall $\tau=0.221$ ), and this increase in $\mathrm{SST}_{100}$ since $4.2 \mathrm{ka}$ is of an average order of $0.8^{\circ} \mathrm{C}$ (obtained by inserting the top and bottom levels in this interval into the regression function).

The REDFIT procedure (Schulz and Mudelsee, 2002) was used to recognize cyclical patterns in the generated $\mathrm{SST}_{100}$ timeseries, and test the significance of peaks in the amplitude spectrum against a nullhypothesis of a red-noise background. The application of this technique to our Holocene ANN SST record (core MD95-2011) indicates the existence of a climate cycle of 278 years length. The runs test inherent in the REDFIT software indicates that the fluctuations in this timeseries are not consistent with a red-noise process ( $r$-value $=30$, which is within the $5 \%$ acceptance region of 24-41) and, as this spectral peak lies above the $98.4 \%$ false-alarm level (Thomson, 1990), the 278-year long cycle in this timeseries is statistically significant against the red-noise background.

\section{Discussion}

\subsection{Spectral analysis of the Holocene ANN-SST record}

Changes in solar activity during the Holocene have an influence over climate at millennial and centennial time scales, as it has been convincingly demonstrated for the North Atlantic (Bond et al., 1997; deMenocal et al., 2000; Bond et al., 2001). One important manifestation of solar variability is the de Vries or Suess cycle (Suess, 1965), which has an average period of
210 years, with a range of $180-220$ years. This cycle has been identified in a variety of settings (Yu and Ito, 1999; Neff et al., 2001), including tree rings (Scuderi, 1993), lake sediments (Anderson, 1992), ice-core records (Raisbeck et al., 1990), and a marine sediment record from the Caribbean Sea (Nyberg et al., 2001). A ca. 200-year cyclicity has also been recognized in the high-resolution oceanic sediment record of Palmer Deep, Antarctic Peninsula, covering the last $13 \mathrm{ka}$ (Domack et al., 2001). This sub-millennial variability is particularly well developed in the late Holocene and is suggested to be connected, in Palmer Deep, to cycles in productivity and Sea Surface Temperature (Leventer et al., 1996), and to solar cycles (Domack et al., 2001). Such a cycle has also been found in Lake Saki (Currie, 1995) and Pakistanian lake varves. Spectral analysis on the MD95-2011 timeseries using the REDFIT procedure (Schulz and Mudelsee, 2002), the most rigorous available for unequally sampled series, instead indicates the existence of a climate cycle of 278 years length, which is close to the de Vries or Suess cycle (Fig. 4). A significant Holocene periodicity of 230 years has been recently documented (Sarnthein et al., 2003) in a core (GeoB23258) from off the western Barents Sea shelf. This core is located along the path of the Western Spitzbergen Current (the northward extension of the Norwegian Current), and its climatic record is therefore presumably affected by most of the climatic mechanisms that are also active in our study area. Our study lends therefore support to the existence of a strong Holocene cyclicity in the Nordic Seas, with a characteristic signature for a period close to the Suess band. The recognized 278year cyclicity is however separated from the Suess cycle, and it has also been reported (Damon and Sonett, 1989) from ${ }^{14} \mathrm{C}$ activity records, and linked to solar variability. Modelling of the solar irradiance forcing also suggests the presence of a cyclicity having a period of ca. 250 years (Weber et al., 2004). Climatic mechanisms associated to a cycle of such length are however, until today, unknown.

\subsection{Downcore predictions: water column gradients?}

We do not observe major differences between the ANN and IKTF methods (Fig. 5), and the remarkably lower SSTs values reconstructed by the ANNs compared to IKTF are easily accounted for, as the former 


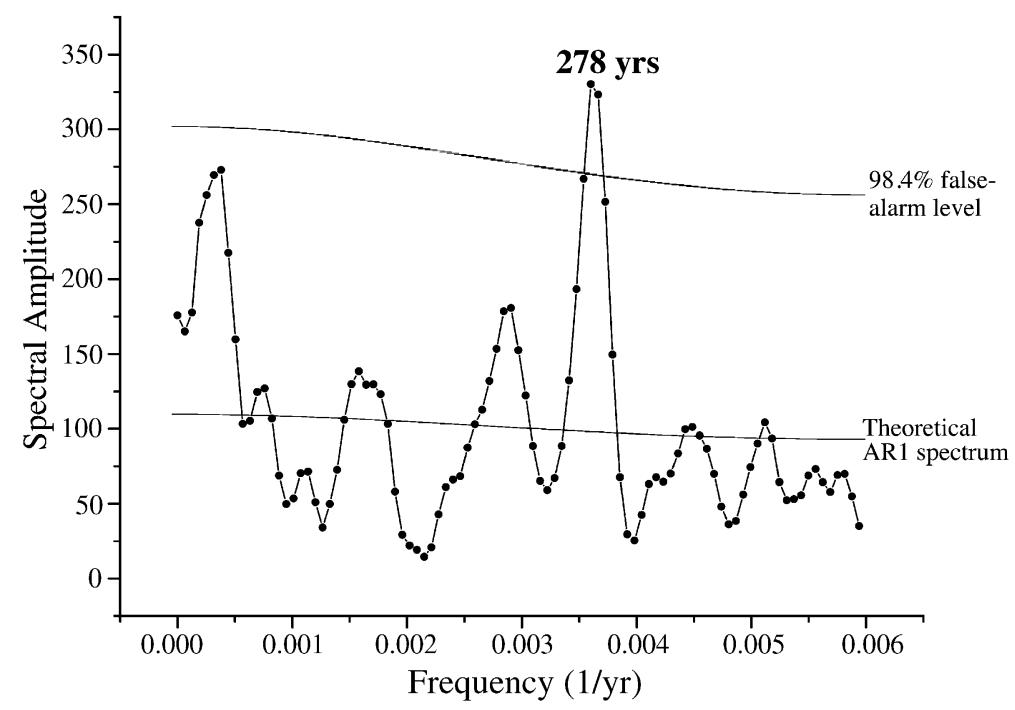

Fig. 4. Amplitude spectrum for the Holocene ANN SST record (core MD95-2011) based on the REDFIT procedure (Schulz and Mudelsee, 2002). One spectral peak at a frequency of 0.0036 above the $98.4 \%$ false-alarm level (Thomson, 1990) suggests the existence of a 278 -year long cycle in this timeseries that is statistically significant against the red-noise background. The theoretical red-noise (AR [1]) spectrum is also shown.

refers to $100 \mathrm{~m}\left(\mathrm{SST}_{100}\right)$, while the latter refers to $0 \mathrm{~m}$ water depth $\left(\mathrm{SST}_{0}\right)$. The good match in the details and in the millennial-scale variability of the two curves witnesses the reliability of both IKTF and ANN at predicting SST in the study area. The main difference consists in a slightly faster cooling in $\mathrm{ANN} \mathrm{SST}_{100}$

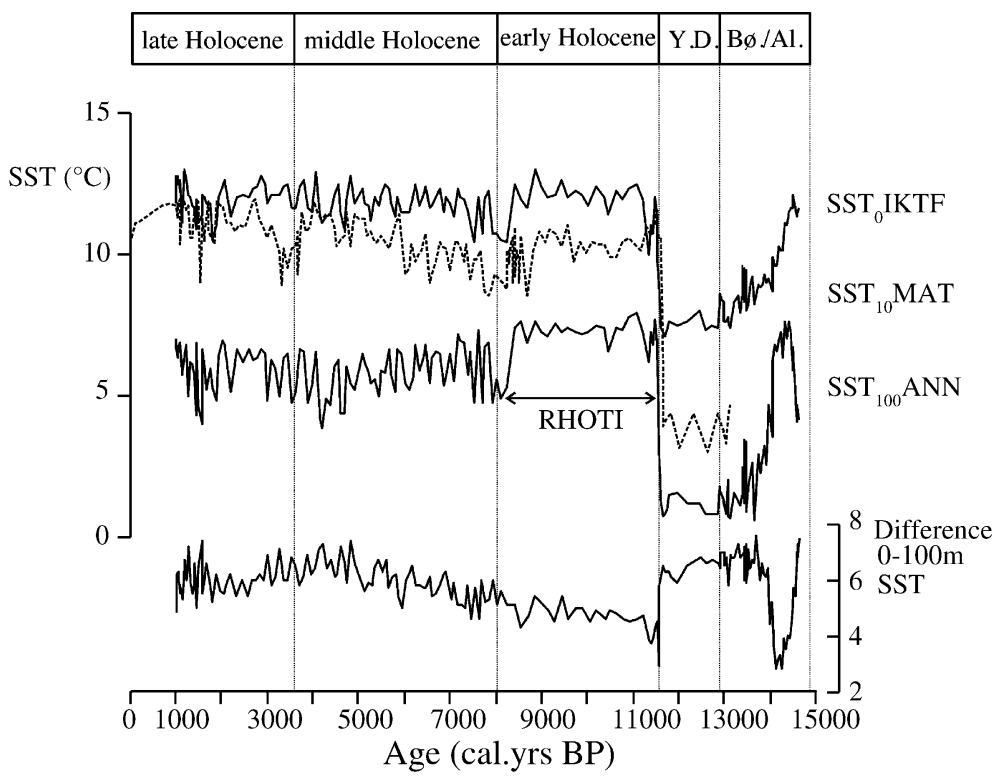

Fig. 5. Comparison between $\mathrm{SST}_{100}$, reconstructed by ANN (this paper) and $\mathrm{SST}_{0}$, reconstructed by the IKTF method (Dolven et al., 2002) for cores MD95-2011 and HM79-4. The difference between the two, representing the degree of mixing of the surface layer, is also shown, along with the Radiolarian Holocene Optimum Temperature Interval (RHOTI), roughly coinciding with the early Holocene. The chronozones are as for Fig. 3. The planktonic foraminifera MAT-derived SST $_{10}$ (Risebrobakken et al., 2003) from core MD95-2011, on the GISP-synchronized time scale, is reported too. 
compared to IKTF $\mathrm{SST}_{0}$ from 14.3 to $13.7 \mathrm{ka}$ (Fig. 5). This could represent a threshold response of the deeper mixed-layer waters to the more gradual cooling trend displayed by the shallower mixed-layer waters, or result from an artefact of the methods.

Based on the present results from ANN ( $\left.\mathrm{SST}_{100}\right)$ and previously published results for $\mathrm{SST}_{0}$ (Dolven et al., 2002), it is possible to compare the temperature evolution from the Bølling/Allerød to present at two different levels in the water column, and to get an idea on the degree of mixing of the surface layer of the ocean (Fig. 5). A trend towards good mixing characterizes the first half of the Bølling/Allerød, while the second half of the Bølling/Allerød and the Younger Dryas are indicative of well-stratified surface layer conditions, with a ca. $7{ }^{\circ} \mathrm{C}$ SST difference between 100 and $0 \mathrm{~m}$ (Fig. 5). The transition to interglacial conditions in the early Holocene marks also the return to well-mixed surface waters, with a difference of only $3{ }^{\circ} \mathrm{C}$ between the two depth levels considered here. During the early and middle Holocene (until ca. $4.2 \mathrm{ka}$ ) one notices a trend towards progressively more stratified waters, mainly connected to the cooling of the deeper $(100 \mathrm{~m})$ waters, while conditions close to the surface remain relatively stable and warm. See next paragraphs for additional discussion over SST trends during the Holocene.

\subsection{Comparison to other late Pleistocene-Holocene paleoclimatic records}

Modern Analog Technique (MAT)-derived SSTs, based on planktonic foraminifera from the MD952011 core, have recently been published (Risebrobakken et al., 2003). These results are very close to our previously published $\mathrm{SST}_{0}$ based on IKTF (Dolven et al., 2002), not indicating particularly warm $\mathrm{SST}_{0}$ during the early Holocene, but displaying relatively stable early Holocene (11.6-8 ka) $\mathrm{SST}_{0}$. The latter gives way to an increasing trend in $\mathrm{SST}_{0}$ into the middle Holocene (from 8 to $4 \mathrm{ka}$ ), and a subsequent very slow warming trend through the late Holocene (3.6-0 ka). Our ANN-based paleo-SST 100 results do not display the cooling trend through the Holocene that was noted in other high-latitude North Atlantic sites (Marchal et al., 2002), and in an Indian Ocean core (Naidu and Malmgren, 2005). In our record, this cooling trend is restricted to the middle Holocene. The two most obvious differences between the ANN-SSTs and MAT-SSTs concern the early Holocene and the middle Holocene, while the two datasets match quite well during the $8.2 \mathrm{ka}$ cooling event. These time intervals and event will be discussed in the following sub-sections.

\subsubsection{The early Holocene (11.6-8.0 ka)}

In order to avoid confusion with the Holocene Climatic Optimum (HCO), taking place in the middle Holocene, from ca. 4 to $7 \mathrm{ka}$, we will refer to the early Holocene radiolarian temperatures, the highest for the Holocene, as the Radiolarian Holocene Optimum Temperature Interval (RHOTI). The presence of an early Holocene RHOTI matches the observation of a maximum in ocean surface temperature off northern Norway between ca. 10.700 and 8.900 cal. years B.P. (Hald et al., 1996), and the calculated post-glacial temperature anomalies in Greenland, which were at a maximum during the Preboreal and Boreal (Johnsen et al., 1995). This is also in accordance with stratigraphic records of the beetle Phratora (Phyllodeca) sp. cf. P. polaris, as this species has at present not been recorded alive in Greenland, while its fossil record shows it was widely distributed in the early Holocene and was undoubtedly favoured by the warmer climate at that time (Bennike et al., 1999). MAT-based SSTs (Risebrobakken et al., 2003) from core MD95-2011 indicate that the early Holocene may be typified by a very slight cooling trend, and its average SST is practically identical to the middle Holocene, and actually colder than the late Holocene. Optimum SSTs are recorded in other SST reconstructions for core MD95-2011, and they occur either between early and middle Holocene, at 9-7 ka, according to diatom-based IKTF (Birks and Koç, 2002), or in the middle Holocene, at 8.5-5.5 ka, as indicated by alkenones (Calvo et al., 2002). Planktic foraminifera, just as radiolarians, may be recording subsurface hydrography changes (Dolven et al., 2002; Risebrobakken et al., 2003). They are therefore imperfect recorders of shallow surface processes and conditions, compared to diatoms and nannoplankton (the latter being the producers of the alkenone signal), which live instead in very shallow water depths. Both diatoms and nannoplankton, however, are themselves affected by problems of another sort as, belonging to the sedimentary fine fraction, may be transported over 
very long distances, and do not always record local SST conditions. Risebrobakken et al. (2003) argue that the lack of high SST during the early Holocene in planktic foraminifera MAT-SSTs, compared to diatom- and alkenone-based records, was caused by an eastward migration of Arctic water, forced by stronger westerlies, which influenced the subsurface water and not the uppermost sea surface water (Blindheim et al., 2000). Hald et al. (1996), however, reported on maximum ocean surface temperatures off northern Norway, based on planktonic foraminifera, during the early Holocene. This, together with the presence of a recognizable RHOTI during the early Holocene in our radiolarian $\mathrm{ANN}$-derived $\mathrm{SST}_{100}$, although not as clear as for diatom- and alkenone-derived SST, questions the theory of stronger westerlies during the early Holocene (Blindheim et al., 2000). These seemingly contradictory results obtained by different fossil groups should not be taken as a negative characteristic of these methods, but represent instead a very promising spinoff, as an integrated approach may be key to the estimation of the degree of water column stratification, which is a very important quantity for the validation and improvement of climate models.

In the case of the Holocene, for instance, the available evidence (HCO presence for nannoplankton, warmer early to middle Holocene for diatoms, warmer early Holocene $\mathrm{SST}_{100}$ for radiolarians, warmer early Holocene ocean surface temperatures for foraminifera) may also indicate a real phenomenon, i.e. a differential behavior of very shallow and subsurface waters through the different Holocene intervals. The early Holocene and the first half of the Bølling/ Allerød would then represent intervals of well-mixed surface layer, with a small contrast between very shallow and $100 \mathrm{~m}$ depths (Fig. 5). During the middle Holocene, on the contrary, an important decoupling between these two layers would take place, and the warming would be limited to the very shallow layers, and not extend down to ca. $100 \mathrm{~m}$ depth. A very strong stratification of the upper water column is present during the second half of the Bølling/Allerød and the whole Younger Dryas, when SSTs are at their minimum. This is probably due to the presence of a cold, low salinity lid, which is commonly associated with the Younger Dryas cooling (Bodén et al., 1997; Spielhagen et al., 1998; Clark et al., 2001). Such a lid could actually be the cause of the Younger Dryas cooling, and model experiments (Manabe and Stouffer, 2000) suggest that the release of $0.1 \mathrm{~Sv}$ of freshwater over 500 years to the North Atlantic, between Greenland and Iceland, results in a drastic cooling (Younger Dryas), followed by a period, just before the Holocene warming, with very unstable ocean temperatures.

\subsubsection{The $8.2 \mathrm{ka}$ cooling event}

This event has been recognized in many paleoclimatic records, and seems to be related to the sudden discharge into the Labrador Sea of freshwater from the periglacial Lake Agassiz (Barber et al., 1999). This localized discharge of low-density waters into very sensitive convection sites for North Atlantic Deep Water formation strongly disturbed the overturning circulation in the North Atlantic, causing its slowdown, and a consequent cooling in the areas around the North Atlantic. During this event, a reduced amount of warm Atlantic water entered the Nordic Seas, as an intermediate current, underlying the lesssalty water originating from the Labrador Sea. This water stratification also probably contributed to the regional North Atlantic cooling by reducing the ocean/atmosphere heat exchange. In our ANN $\mathrm{SST}_{100}$ record, the $8.2 \mathrm{ka}$ cooling event is clearly shown between 8.1 and $8.5 \mathrm{ka}$ (Fig. 6), and represents a $2.7{ }^{\circ} \mathrm{C}$ cooling lasting 420 years. This matches quite well with that recognized in the MAT SST record, i.e. a ca. $3{ }^{\circ} \mathrm{C}$ decrease in SST centered at $8.0-8.1 \mathrm{ka}$ (Risebrobakken et al., 2003). In the northern North Sea, Klitgaard-Kristensen et al. (1998) recognized that $N$. pachyderma (sin.) reaches a $15-16 \%$ abundance peak, against its background 2-7\% abundance during last 10.000 cal. years B.P., and correlated this peak to the Greenland GRIP ice core 8200 years event. Knies et al. (2003) reported two prominent lows in marine organic carbon in Andfjorden that may correlate with the Preboreal Oscillation (PBO; 11.17-11.05 ka) and the Greenland 8.2 ka event, as discussed by Björck et al. (1996) and Alley et al. (1997), respectively. The PBO is probably also present in our data (Fig. 3, Table 3 ), showing a gradual temperature increase over three samples from $6.2{ }^{\circ} \mathrm{C}$ (11.370 cal. years B.P.) till $7.9{ }^{\circ} \mathrm{C}$ (11.074 cal. years B.P.). Klitgaard-Kristensen et al. (1998) concluded that the synchronous timing of the cooling event in the Greenland icecores, marine records and tree ring data from north- 


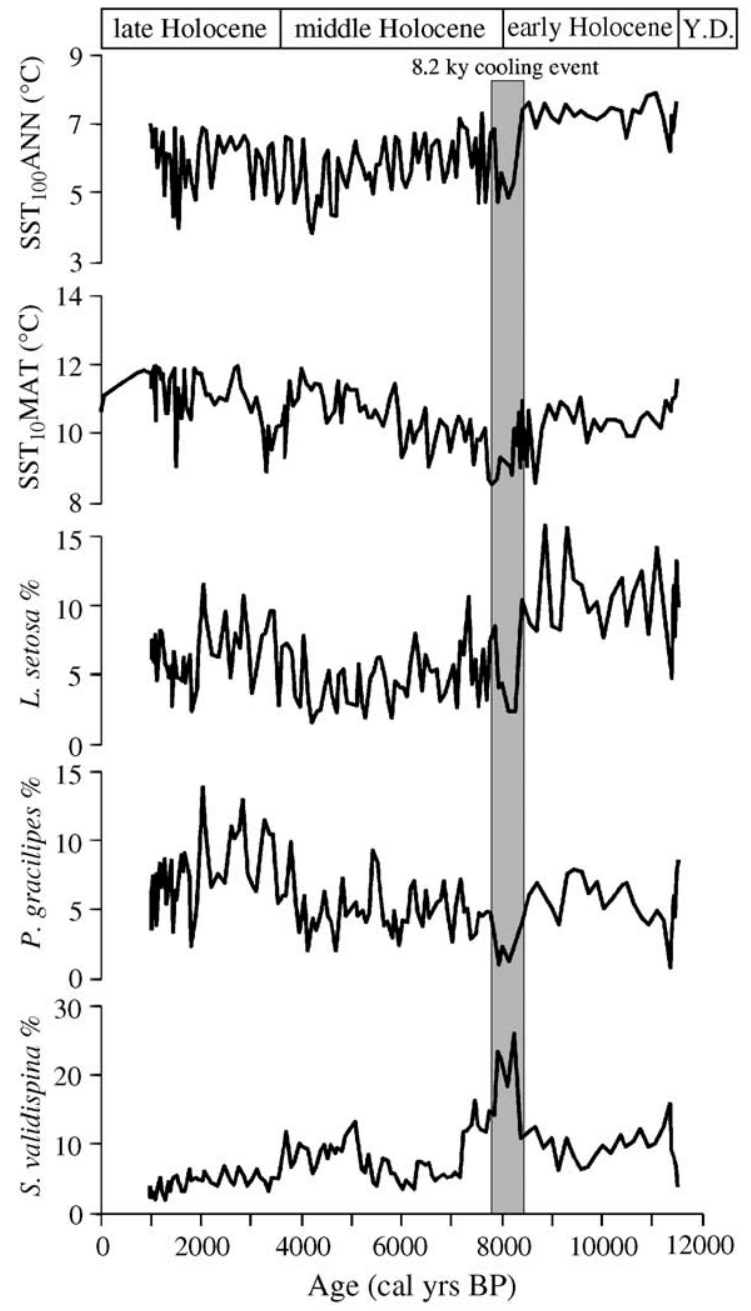

Fig. 6. Holocene changes in ANN-derived $\mathrm{SST}_{100}$, and corresponding variations in the relative abundances of three radiolarian species (L.setosa, P. gracilipes, and S. validispina). The $8.2 \mathrm{ka}$ cooling event is shaded for reference. Y.D.=Younger Dryas. The planktonic foraminifera MAT-derived $\mathrm{SST}_{10}$ (Risebrobakken et al., 2003) from core MD95-2011, on the GISP-synchronized time scale, is reported too.

west Europe suggests a regional influence on the North Atlantic ocean-atmosphere system and the thermohaline circulation.

The radiolarian assemblage changes associated with the $8.2 \mathrm{ka}$ cooling event (Fig. 6) include a major decrease in the relative abundance of Lithomelissa setosa and Pseudodictyophimus gracilipes, while Stylodictya validispina displays an absolute maximum during this time interval. The first two species are main components of the warm water assemblage (Factor 4) recognized in the study area (Cortese et al., 2003). Their modern distribution is either very strongly linked to the warm Norwegian Current (L. setosa), or centered around the eastern boundary of the Arctic Front (P. gracilipes), in the gyre east of Jan Mayen/Iceland Plateau, i.e. they are found in waters warmer than those present today on the Iceland Plateau (Cortese et al., 2003). According to their modern distribution, it seems that the radiolarian assemblage change during the $8.2 \mathrm{ka}$ event was represented rather by a decrease in warm water species, than by an increase in cold ones. This may indicate a reduction of the importance of the warm Norwegian Current in the study area, a consequence of the slowdown of the North Atlantic overturning circulation. The latter is, as mentioned earlier, a result of freshwater discharges from periglacial lakes around the Laurentide ice sheet, the current explanation for the 8.2 ka cooling (Barber et al., 1999; Alley et al., 1997). The shift in abundance of $S$. validispina (from $11 \%$ to $26 \%$, Fig. 6) may also be a response to this meltwater flux, as closely related representatives of the family Spongodiscidae, i.e. Spongotrochus glacialis, Spongaster tetras, Stylochlamydium venustum have been demonstrated (Boltovskoy, 1998; Nimmergut and Abelmann, 2002) to live in the shallowest part of the water column, and to be able to adapt to a broad range of temperature and salinity conditions. In the Sea of Okhotsk, for example, sea ice melt has a strong impact, and a low-salinity layer develops at the surface during the spring-summer melt season. In the Sea of Okhotsk, juvenile Spongodiscidae display an abundance peak in the surface layer (Nimmergut and Abelmann, 2002), and are almost the only radiolarians capable of thriving in such low salinity conditions. Blueford (1983) also demonstrated that juvenile and adult Spongodiscidae were dominant in the surface sediments of the shallow northeastern parts of the Bering Sea, an area also influenced by low salinity conditions.

\subsubsection{The middle $(8.0-3.6 \mathrm{ka})$ and late $(3.6-0 \mathrm{ka})$ Holocene}

The SST reconstructions for the 8-3.6 ka interval, roughly corresponding to the middle Holocene, are substantially different between ANN SST 100 (showing a cooling trend) and MAT SST, indicating a warming 
trend (Risebrobakken et al., 2003). The latter, however, is difficult to reconcile with the abundances of $N$. pachyderma sinistral morphotype during the middle Holocene, which should be indicative of a relatively constant, or even slightly cooling trend. The presence of a cooling trend is also supported by the maximum values reached by Globigerina quinqueloba during the middle Holocene, indicative of repeated Arctic water influence into the Norwegian Sea at this time, as this species is associated with the Arctic Front area (Johannessen et al., 1994). However, the general Holocene trends of ANN $\mathrm{SST}_{100}$ and MAT SSTs are relatively well comparable. There is an apparent discrepancy between the two methods for the absolute SST values $\left(4-5{ }^{\circ} \mathrm{C}\right.$ difference during the Holocene, 2 ${ }^{\circ} \mathrm{C}$ difference during the Younger Dryas, with MAT recording, for both time intervals, warmer temperatures). This is, however, easily accounted for, as it relates to the different water depth the reconstructed SSTs refer to: $100 \mathrm{~m}$ depth for ANN (this paper), $10 \mathrm{~m}$ depth for MAT (Risebrobakken et al., 2003). In our MD95-2011 record, the late Holocene is characterized by a slight, but statistically significant, warming trend, with ANN SST 100 recovering after the middle Holocene cooling trend. A slight trend towards a lessstratified surface layer is also evident during the late Holocene (Fig. 5), after the maximum Holocene mixed-layer stratification, which was reached during the youngest portion of the middle Holocene.

\section{Summary}

The main aim of this study was to apply Artificial Neural Networks to the reconstruction of surface water temperature in an area that is very sensible to heat transport to high latitudes and to thermohaline overturning. The main results of this study can be so summarized:

- ANN have been successfully calibrated in the Nordic Seas, with both high correlation coefficients between observed and predicted values $(r>0.95$, but for one case) and satisfactorily low RMSEPs $\left(0.62-1.00{ }^{\circ} \mathrm{C}\right)$, which allows the reconstruction of past SSTs within relatively low error limits;

- Optimal correlation is found for the summer temperature at $100 \mathrm{~m}$ depth, indicating how this season and depth are particularly important for the formation of the radiolarian assemblage signal in the study area at present;

- The first half of the Bølling/Allerød and the early Holocene represent time intervals when $\mathrm{SST}_{100}$ reached a maximum;

- The Preboreal Oscillation (PBO) and the Greenland $8.2 \mathrm{ka}$ event are both present in our record. There is a drop of $1.7{ }^{\circ} \mathrm{C}$ and a rise of $1.1^{\circ} \mathrm{C}$ in and out of the PBO, respectively, while the $8.2 \mathrm{ka}$ event is represented by a $2.7^{\circ} \mathrm{C}$ cooling lasting 420 years;

- The middle Holocene is characterized by a cooling trend in $\mathrm{SST}_{100}$, while the late Holocene displays a warming trend;

- The Holocene $\mathrm{SST}_{100}$ signal includes a strong cyclicity (278 years period) close to the Suess band;

- There is good agreement between the ANN and IKTF-derived SSTs: the main difference is represented by a faster cooling in $\mathrm{ANN} \mathrm{SST}_{100}$ compared to IKTF $\mathrm{SST}_{0}$ during the Bølling/Allerød;

- MAT-derived SSTs based on planktonic foraminifera also match well our reconstructions, with the exception of the absence of a SST optimum during the early Holocene, and a warming trend during the middle Holocene;

- The highest SSTs during the Holocene occur at different times and depths according to different methods and fossil groups (underlined $=$ our data, evidence from other methods cited in the Discussion paragraph): early Holocene ( $S S T_{100}$, radiolarians, ANN), between early and middle Holocene ( $\mathrm{SST}_{0}$, diatoms, IKTF), middle Holocene $\left(\mathrm{SST}_{0}\right.$, alkenones). Other methods do not indicate the presence of a clear SST optimum during the Holocene $\left(\mathrm{SST}_{10}\right.$, planktonic foraminifera, MAT; $S S T_{0}$, radiolarians, $I K T F$ ). This information can be used to reconstruct the degree of mixing of the upper water column.

\section{Acknowledgements}

Dave Lazarus and an anonymous reviewer are thanked for their suggestions and improvements to the manuscript. We warmly thank Øyvind Hammer for reading and commenting an earlier version of the manuscript, which was revised according to his 
remarks. Sincere thanks go to Björg Risebrobakken who generously shared her data. G. Cortese was supported, during the realization of this paper, by the Deutsche Forschungsgemeinschaft as part of the DFG-Research Center "Ocean Margins" of the University of Bremen, No. RCOM0288. The University of Oslo and the Norwegian Research Council funded J.K. Dolven when producing the radiolarian data from HM79-4 and MD95-2011.

\section{References}

Abelmann, A., Brathauer, U., Gersonde, R., Sieger, R., Zielinski, U., 1999. Radiolarian-based transfer function for the estimation of sea surface temperatures in the Southern Ocean (Atlantic sector). Paleoceanography 14 (3), 410-421.

Alley, R.B., Mayewski, P.A., Sowers, T., Stuiver, M., Taylor, K.C., Clark, P.U., 1997. Holocene climatic instability: a prominent widespread event 8200 years ago. Geology 25, 483-486.

Anderson, R.Y., 1992. Possible connections between surface winds, solar activity, and the earth's magnetic field. Nature 358, 51-53.

Barber, D.C., Dyke, A., Hillaire-Marcel, C., Jennings, A.E., Andrews, J.T., Kerwin, M.W., Bilodeau, G., McNeely, R., Southon, J., Morehead, M.D., Gagnonk, J.-M., 1999. Forcing of the cold event of 8200 years ago by catastrophic drainage of Laurentide lakes. Nature 400, 344-348.

Bennike, O., Björck, S., Böcher, J., Hansen, L., Heinemeier, J., Wohlfahrt, B., 1999. Early Holocene plant and animal remains from North-East Greenland. Journal of Biogeography 26, 667-677.

Birks, H.J.B., 1995. Quantitative palaeoenvironmental reconstructions. In: Maddy, D., Brew, J.S. (Eds.), Statistical Modeling of Quaternary Science Data. Quaternary Research Association, Cambridge, U.K., pp. 161-254.

Birks, H.H., Gulliksen, S., Haflidason, H., Mangerud, J., 1996. New radiocarbon dates for the Vedde Ash and the Saksunarvatn Ash from western Norway. Quaternary Research 45, 119-127.

Birks, C.J.A., Koç, N., 2002. A high-resolution diatom record of late-Quaternary sea-surface temperatures and oceanographic conditions from the eastern Norwegian Sea. Boreas 31, $323-344$.

Björck, S., Kromer, B., Johnson, S., Bennike, O., Hammarlund, D., Lemdahl, G., Possnert, G., Rasmussen, T.L., Wohlfahrt, B., Hammer, C.U., Spurk, M., 1996. Synchronized terrestrial-atmospheric deglacial records around the North Atlantic. Science 274, 1155-1160.

Bjørklund, K.R., 1974. The seasonal occurrence and depth zonation of radiolarians in Korsfjorden, Western Norway. Sarsia 56, $13-42$.

Bjørklund, K.R., Cortese, G., Swanberg, N., Schrader, H.J., 1998. Radiolarian faunal provinces in surface sediments of the Greenland, Iceland and Norwegian (GIN) Seas. Marine Micropaleontology $35,105-140$.
Blindheim, J., Borokov, V., Hansen, B., Malmberg, S.A., Turrell, W.R., Østerhus, S., 2000. Upper layer cooling and freshening in the Norwegian Sea in relation to atmospheric forcing. Deep Sea Research, Part I 47, 655-680.

Blueford, J.R., 1983. Distribution of Quaternary radiolaria in the Navarin Basin geologic province, Bering Sea. Deep-Sea Research 30 (7A), 763-781.

Bodén, P., Fairbanks, R.D., Wright, J.D., Burckle, L.H., 1997. High-resolution stable isotope records from southwest Sweden: the drainage of the Baltic Ice Lake and Younger Dryas ice margin oscillations. Paleoceanography 12, 39-49.

Boltovskoy, D., 1998. Classification and distribution of South Atlantic recent polycystine radiolaria. Paleontologica Electronica 1 (2).

Bond, G., Showers, W., Cheseby, M., Almasi, P., deMenocal, P., Priore, P., Hajdas, I., Bonani, G., 1997. A pervasive millennialscale cycle in North Atlantic Holocene and glacial climates. Science 278, 1257-1266.

Bond, G., Kromer, B., Beer, J., Muscheler, R., Evans, M.N., Showers, W., Hoffmann, S., Lotti-Bond, R., Hajdas, I., Bonani, G., 2001. Persistent solar influence on North Atlantic climate during the Holocene. Science 294, 2130-2136.

Calvo, E., Grimalt, J., Jansen, E., 2002. High resolution $U_{37}^{K}$ sea surface temperature reconstruction in the Norwegian Sea during the Holocene. Quaternary Science Reviews 21, $1385-1394$.

Clark, P.U., Marshall, S.J., Clarke, G.K.C., Hostetler, S., Licciardi, J.M., Teller, J.T., 2001. Freshwater forcing of abrupt climate change during the last glaciation. Science 293, 283-287.

Conkright, M., Levitus, S., O’Brien, T., Boyer, T., Antonov, J., Stephens, C., 1998. World Ocean Atlas 1998 CD-ROM Data Set Documentation, National Oceanographic Data Center (NODC) Internal Report, Silver Spring, Maryland.

Cortese, G., Abelmann, A., 2002. Radiolarian-based paleotemperatures during the last $160 \mathrm{kyrs}$ at ODP Site 1089 (Southern Ocean, Atlantic Sector). Palaeogeography, Palaeoclimatology, Palaeoecology 182 (3-4), 259-286.

Cortese, G., Bjørklund, K.R., Dolven, J.K., 2003. Polycystine radiolarians in the Greenland-Iceland-Norwegian Seas: species and assemblage distribution. Sarsia 88, 65-88.

Currie, R.G., 1995. Luni-solar and solar cycle signals in Lake Saki varves and further experiments. International Journal of Climatology $15(8), 893$.

Damon, P.E., Sonett, C.P., 1989. Solar and terrestrial components of the atmospheric ${ }^{14} \mathrm{C}$ variation spectrum. In: Sonett, C.P. (Ed.), The Sun in Time. Univ. Arizona Press, Tucson, AZ.

deMenocal, P., Ortiz, J., Guilderson, T., Sarnthein, M., 2000. Coherent high- and low-latitude climate variability during the Holocene warm period. Science 288, 2198-2202.

Dolven, J.K., Bjørklund, K.R., 2001. An early Holocene peak occurrence and recent distribution of Rhizoplegma boreale (Radiolaria): a biomarker in the Norwegian Sea. Marine Micropaleontology 42, 25-44.

Dolven, J.K., Cortese, G., Bjørklund, K.R., 2002. A high-resolution radiolarian-derived paleotemperature record for the Late Pleistocene-Holocene in the Norwegian Sea. Paleoceanography 17 (4), 1072. doi:10.1029/2002PA000780. 
Domack, E., Leventer, A., Dunbar, R., Taylor, F., Brachfeld, S., Sjunneskog, C., ODP Leg 178 Scientific Party, 2001. Chronology of the Palmer Deep site, Antarctic Peninsula: a Holocene palaeoenvironmental reference for the circum-Antarctic. The Holocene 11, 1-9.

Ganopolski, A., Rahmstorf, S., 2001. Rapid changes of glacial climate simulated in a coupled climate model. Nature 409, $153-158$.

Hald, M., Dokken, T., Hagen, S., 1996. Paleoceanography on the European Arctic Margin during the last deglaciation. In: Andrews, J.T., Austin, W.E.N., Bergsten, H., Jennings, A.E., et al., (Eds.), The Quaternary Paleoceanography of the North Atlantic Margins, 111. Geological Society Special Publication, pp. $275-287$.

Hutson, W.H., 1979. The Agulhas Current during the late Pleistocene: analysis of modern faunal analogs. Science 207, 64-66.

Imbrie, J., Kipp, N.G., 1971. A new micropaleontological method for quantitative paleoclimatology: application to a late Pleistocene Caribbean core. In: Turekian, K. (Ed.), Late Cenozoic Glacial Ages. Yale University Press, New Haven, Connecticut, pp. $71-181$.

IPCC, 2001. Climate change 2001: the scientific basis. Contribution of Working Group I to the Third Assessment Report of the Intergovernmental Panel on Climate Change. Cambridge University Press, Cambridge, United Kingdom. 881 pp.

Johannessen, T., Jansen, E., Flatøy, A., Ravelo, A.C., 1994. The relationship between surface water masses, oceanographic fronts and paleoclimatic proxies in surface sediments of the Greenland, Iceland and Norwegian Seas. In: Zahn, R., Kaminski, M., Labeyrie, L. (Eds.), Carbon Cycling in Glacial Ocean: Constraints on the Ocean's Role in Global Change, NATO ASI Series. Springer-Verlag, New York, pp. 61-85.

Johnsen, S.J., Dahl-Jensen, D., Dansgaard, W., Gundestrup, N., 1995. Greenland palaeotemperatures derived from GRIP bore hole temperature and ice core isotope profiles. Tellus 47B, 624-629.

Kendall, M., Ord, J.K., 1990. Time Series, 3rd ed. Edward Arnold. 296 pp.

Klitgaard-Kristensen, D., Sejrup, H.P., Haflidason, H., Johnsen, S., Spurk, M., 1998. A regional 8200 cal. yr BP cooling event in northwest Europe, induced by final stages of the Laurentide ice-sheet deglaciation? Journal of Quaternary Science 13 (2), $165-169$.

Knies, J., Hald, M., Ebbesen, H., Mann, U., Vogt, C., 2003. A deglacial - middle Holocene record of biogenous sedimentation and paleoproductivity changes from the northern Norwegian shelf. Paleoceanography 18 (4), 1096. doi:10.1029/ 2002PA000872.

Koc-Karpuz, N., Jansen, E., 1992. A high-resolution diatom record of the last deglaciation from the SE Norwegian Sea: documentation of rapid climatic changes. Paleoceanography 7, 499-520.

Leventer, A., Domack, E.W., Ishman, E., Brachfeld, S., McClennen, C.E., Manley, P., 1996. Productivity cycles of 200-300 years in the Antarctic Peninsula region: understanding linkages among the sun, atmosphere, oceans, sea ice, and biota. Geological Society of America Bulletin 108, 1626-1644.
Malmgren, B.A., Nordlund, U., 1997. Application of artificial neural networks to paleoceanographic data. Palaeogeography, Palaeoclimatology, Palaeoecology 136, 359-373.

Manabe, S., Stouffer, R.J., 2000. Study of abrupt climate change by a coupled ocean-atmosphere model. Quaternary Science Reviews 19, 285-299.

Mangerud, J., Anderson, S.T., Berglund, B.E., Donner, J., 1974. Quaternary stratigraphy of Norden, a proposal for terminology and classification. Boreas 3, 109-128.

Marchal, O., Cacho, I., Stocker, T.F., Grimalt, J.O., Calvo, E., Martrat, B., Shackleton, N., Vautravers, M., Cortijo, E., van Kreveld, S., Andersson, C., Koç, N., Chapman, M., Sbaffi, L., Duplessy, J.C., Sarnthein, M., Turon, J.L., Duprat, J., Jansen, E., 2002. Apparent long-term cooling of the sea surface in the northeast Atlantic and Mediterranean during the Holocene. Quaternary Science Reviews 21, 455-483.

McCulloch, W.S., Pitts, W., 1943. A logical calculus of the idea immanent in nervous activity. Bulletin of Mathematical Biophysics $5,115-133$.

Molina-Cruz, A., 1984. The radiolarian remains as indicators of upwelling processes: the Peruvian connection. Marine Micropaleontology $9,53-75$.

Moore Jr., T.C., 1973. Late Pleistocene-Holocene oceanographic changes in the northeastern Pacific. Quaternary Research 3 (1), 99- 109 .

Morley, J.J., 1979. A transfer function for estimating paleoceanographic conditions, based on deep-sea surface distribution of radiolarian assemblages in the South Atlantic. Quaternary Research 12, 381-395.

Naidu, P.D., Malmgren, B.A., 2005. Seasonal Sea Surface Temperature contrast between Holocene and Last Glacial Maximum in the Arabian Sea: modulated by monsoon upwelling. Paleoceanography 20, PA1004. doi:10.1029/2004PA001078.

Neff, U., Burns, S.J., Mangini, A., Mudelsee, M., Fleitmann, D., Matter, A., 2001. Strong coherence between solar variability and the monsoon in Oman between 9 and 6 kyr ago. Nature 411, 290-293.

Nimmergut, A., Abelmann, A., 2002. Spatial and seasonal changes of radiolarian standing stocks in the Sea of Okhotsk. Deep-Sea Research I 49, 463-493.

Nyberg, J., Kuijpers, A., Malmgren, B.A., Kunzendorf, H., 2001. Late Holocene changes in precipitation and hydrography recorded in marine sediments from the northeastern Caribbean Sea. Quaternary Research 56, 87-102.

Pflaumann, U., Duprat, J., Pujol, C., Labeyrie, L., 1996. SIMMAX: a modern analog technique to deduce Atlantic sea surface temperatures from planktonic foraminifera in deep-sea sediments. Paleoceanography 11, 15-35.

Pisias, N.G., Roelofs, A., Weber, M., 1997. Radiolarian-based transfer functions for estimating mean surface ocean temperatures and seasonal range. Paleoceanography 12 (3), 365-379.

Raisbeck, G.M., Yiou, F., Jouzel, J., Petit, J.R., 1990. ${ }^{10}$ Be and ${ }^{2} \mathrm{H}$ in polar ice cores as a probe of the solar variability's influence on climate. Philosophical Transactions of the Royal Society of London. A 330, 463-470.

Risebrobakken, B., Jansen, E., Andersson, C., Mjelde, E., Hevrøy, K., 2003. A high-resolution study of Holocene paleoclimatic 
and paleoceanographic changes in the Nordic Seas. Paleoceanography 18 (1), 1017. doi:10.1029/2002PA000764.

Sarnthein, M., Van Kreveld, S., Erlenkeuser, H., Grootes, P.M., Kucera, M., Pflaumann, U., Schulz, M., 2003. Centennial-tomillennial-scale periodicities of Holocene climate and sediment injections off the western Barents shelf, 75 degrees N. Boreas 32 (3), $447-461$.

Schlitzer, R., 2000. Ocean Data View. http://www.awi-bremerhaven. $\mathrm{de} / \mathrm{GEO} / \mathrm{ODV}$.

Schröder-Ritzrau, A., 1995. Aktuopaläontologische Untersuchung $\mathrm{zu}$ Verbreitung und Vertikalfluss von Radiolarien sowie ihre räumliche und zeitliche Entwicklung im Europäischen Nordmeer. Ph.D. Thesis, Universität zu Kiel.

Schulz, M., Mudelsee, M., 2002. REDFIT: estimating red-noise spectra directly from unevenly spaced paleoclimatic time series. Computers and Geosciences 28, 421-426.

Scuderi, L.A., 1993. A 2000-year tree ring record of annual temperatures in the Sierra Nevada Mountains. Science 259, $1433-1436$.

Spielhagen, R.F., Nørgaard-Pedersen, N., Erlenkauser, H., Grootes, P.M., Heinemeier, J., 1998. A meltwater event in the Arctic Ocean before the Younger Dryas. Paper presented at the 6th International Conference on Paleoceanography, UNESCO, Lisbon, 23-28 August.
Suess, H.E., 1965. Secular variations of the cosmic-ray-produced Carbon-14 in the atmosphere and their interpretation. Journal of Geophysical Research 70, 5937-5952.

ter Braak, C.J.F., 1986. Canonical correspondence analysis: a new eigenvector technique for multivariate direct gradient analysis. Ecology 67, 1167-1179.

ter Braak, C.J.F., Juggins, S., 1993. Weighted averaging partial least squares regression (WA-PLS): an improved method for reconstructing environmental variables from species assemblages. Hydrobiologia 269-270, 485-502.

Thomson, D.J., 1990. Time series analysis of Holocene climate data. Philosophical Transactions of the Royal Society of London. Series A 330, 601-616.

Waelbroeck, C., Labeyrie, L.D., Duplessy, J.C., Guiot, J., Labracherie, M., Leclaire, H., Duprat, J., 1998. Improving past sea surface temperature estimates based on planktonic fossil faunas. Paleoceanography 13 (3), 272-283.

Weber, S.L., Crowley, T.J., van der Schrier, G., 2004. Solar irradiance forcing of centennial climate variability during the Holocene. Climate Dynamics 22 (5), 539-553.

Yu, Z., Ito, E., 1999. Possible solar forcing of century-scale drought frequency in the northern Great Plains. Geology 27, 263-266. 